\title{
On Perturbations of Unitary Minimal Models by Boundary Condition Changing Operators
}

\author{
K. Graham円 \\ December 6, 2018 \\ Mathematics Department, \\ King's College London, Strand, London WC2R 2LS, U.K.
}

\begin{abstract}
In this note we consider boundary perturbations in the A-Series unitary minimal models by $\phi_{r, r+2}$ fields on superpositions of boundaries. In particular, we consider perturbations by boundary condition changing operators. Within conformal perturbation theory we explicitly map out the space of perturbative renormalisation group flows for the example $\phi_{13}$ and find that this sheds light on more general $\phi_{r, r+2}$ perturbations. Finally, we find a simple diagrammatic representation for the space of flows from a single Cardy boundary condition.
\end{abstract}

\section{Introduction}

The study of boundary perturbations in rational conformal field theory has found applications in many areas of string theory, condensed matter physics and statistical mechanics. Tachyon condensation and the Kondo problem being but two. Previous investigations of boundary perturbations have concentrated on systems with integrability where there are many powerful techniques available. More general studies began with [3, 2] and were continued in [14, 1, 10]. In these latter works, the integral role of superpositions of boundaries came to the fore and has provided the impetus for the present work.

In this note we consider boundary perturbations in the unitary A-series Virasoro minimal models by $\phi_{r, r+2}$ fields on a superposition of boundary conditions. In particular this includes perturbations by boundary condition changing operators. The paper begins with an introduction to the Virasoro minimal models, their boundaries and their boundary condition changing operators (henceforth called simply "boundary changing operators"). Then in section 3 we look at the general theory of $\phi_{r, r+2}$ perturbations within perturbation theory, illustrating it with two examples: $\phi_{13}$ boundary condition changing perturbations and $\phi_{35}$ perturbations on the boundary condition $(2, p), p>2$. Here we are led to consider perturbations by irrelevant operators. In section 3.2 we find the fixed points of our example systems before identifying the associated boundary theories in sections 1 and 5 . There is an intriguing relation between our flows and the lattice model studies of Behrend and Pearce [4]. The relation takes the form of a simple set of diagrammatic rules that we claim, describe the space of renormalisation

\footnotetext{
${ }^{1}$ e-mail: kgraham@mth.kcl.ac.uk
} 
group flows for a general Cardy boundary condition. This is the subject of section 6. We conclude with a discussion and directions for future research.

\section{Unitary Minimal Models and their Boundaries}

This section reviews a few facts about the unitary A-series Virasoro minimal model $M_{m, m+1}$, with central charge $c=1-\frac{6}{m(m+1)}$. The primary fields of the bulk theory are characterised by their conformal weight,

$$
h_{r, s}=\frac{1}{4 m(m+1)}\left[(r(m+1)-s m)^{2}-1\right],
$$

where the two integers $r$ and $s$ are taken from the Kac table, $(r, s) \in K=\left\{\left(r^{\prime}, s^{\prime}\right): 1 \leq r^{\prime} \leq\right.$ $\left.m-1,1 \leq s^{\prime} \leq m\right\}$ modulo the symmetry $(r, s) \rightarrow(m-r, m+1-s)$. We define $K^{\prime} \subset K$ such that only one of each pair $\{(r, s),(m-r, m+1-s)\}$ is included in $K^{\prime}$.

All conformal boundary conditions can be built out of so-called "Cardy" boundary conditions [7] which are defined to have a non-degenerate vacuum sector. Each Cardy boundary condition is in one-to-one correspondence with a primary field of the bulk theory and so are also labeled by the Kac table. A general boundary condition is then a linear combination of Cardy boundary conditions with positive integer coefficients.

The spectrum of boundary fields on a general boundary condition $a=\oplus_{i=1}^{n} a_{i}, \quad a_{i}=$ $\left(r_{i}, s_{i}\right)$, is succinctly represented by the cylinder partition function,

$$
Z_{a a}(q)=\sum_{i, j} Z_{a_{i} a_{j}}(q)=\sum_{\substack{i, j \\ b \in K^{\prime}}} n_{b a_{i}}{ }^{a_{j}} \chi_{b}(q)
$$

where $q=e^{-\pi L / R}, L, R$ are the circumference and length of the cylinder respectively, $\chi_{b}(q)$ is the character of the representation $b$ and $n_{b a_{i}}{ }^{a_{j}}$ are the fusion numbers of the minimal model (see e.g. [5]),

$$
n_{(r, s)\left(r^{\prime}, s^{\prime}\right)}\left(r^{\prime \prime}, s^{\prime \prime}\right)=N(m)_{r, r^{\prime}} r^{\prime \prime} N(m+1)_{s, s^{\prime}} s^{\prime \prime}+N(m)_{m-r, r^{\prime}} r^{\prime \prime} N(m+1)_{m+1-s, s^{\prime}} s^{\prime \prime},
$$

where,

$$
N(m)_{r, r^{\prime}} r^{\prime \prime}= \begin{cases}1 & r+r^{\prime}+r^{\prime \prime} \text { odd, },\left|r-r^{\prime}\right|<r^{\prime \prime} \text { and } r^{\prime \prime}<r+r^{\prime}<2 m-r^{\prime \prime} \\ 0 & \text { otherwise. }\end{cases}
$$

are the fusion coefficients for $\widehat{s u}(2)_{m-2}$.

One of the lessons to take from (2.2) is that when considering superpositions of boundaries, one must also take into account boundary changing operators as an integral part of the theory. For example, consider the infinite strip with boundary conditions $a$ on the left and $b$ on the right. Moving to the disc we see the points at infinity are mapped to a pair of operators inserted on the boundary which have different boundary conditions on either side of them. Rotate the system slightly and then map back to the strip. These insertions are now at a finite distance along the strip and can be seen to act in the Hilbert space of the theory: taking 
elements from the Hilbert space of the strip with boundary conditions $a$ and $a, H_{a a}$ into $H_{a b}$ say. These are boundary changing operators.

A quantity central to the following is the boundary entropy $g$ of Affleck and Ludwig, 3, 2], whose value on a $(r, s)$ boundary condition is given by,

$$
\langle\mathbb{1}\rangle_{(r, s)}=g_{(r, s)}=\left(\frac{8}{m(m+1)}\right)^{\frac{1}{4}} \frac{\sin \left(\frac{\pi r}{m}\right) \sin \left(\frac{\pi s}{m+1}\right)}{\sqrt{\sin \left(\frac{\pi}{m}\right) \sin \left(\frac{\pi}{m+1}\right)}} .
$$

On a superposition, its value is simply the sum of the values of the component Cardy boundaries. We will also require the one point function of primary bulk fields on the (unit) disc,

$$
\left\langle\Phi_{r, s}(z, \bar{z})\right\rangle_{\omega}=\frac{A_{\omega}^{(r, s)}}{|1-z \bar{z}|^{2 h_{r, s}}},
$$

where for a Cardy boundary,

$$
A_{\left(r^{\prime}, s^{\prime}\right)}^{(r, s)}=(-1)^{\left(s^{\prime}+r^{\prime}\right)(r+s)}\left(\frac{8}{m(m+1)}\right)^{\frac{1}{4}} \frac{\sin \left(\frac{\pi r r^{\prime}}{m}\right) \sin \left(\frac{\pi s s^{\prime}}{m+1}\right)}{\sqrt{\sin \left(\frac{\pi r}{m}\right) \sin \left(\frac{\pi s}{m+1}\right)}},
$$

while for a superposition, one again sums the contributions of each component boundary.

Finally we set our conventions for boundary fields and for taking the operator product expansion (OPE). Fields will be defined on a disc of circumference $L$, parameterised by $x \in[0, L)$. We label our boundary fields of weight $h_{i}$ by $\phi_{i}^{a b}(x)$ : such a field interpolates between boundary conditions $a$ in the region $>x$ and $b$ in the region $<x$. The OPE of two boundary fields is given by,

$$
x>y, \quad \phi_{i}^{a b}(x) \phi_{j}^{c d}(y) \sim \sum_{k}(x-y)^{-h_{i}-h_{j}+h_{k}} \delta^{b c} C_{i j}^{(a b d) k} \phi_{k}^{a d}\left(\frac{1}{2}(x+y)\right)+\text { desc. },
$$

where "desc." denotes Virasoro descendants of the primary boundary fields and the structure constants are known from the work of Runkel 15.

\subsection{The $c \rightarrow 1$ limit of Boundary Minimal Models}

We are interested in the boundary minimal models as $c \rightarrow 1, m \rightarrow \infty$ and so collect here a couple of important results about the limit. From the partition function (2.2) we see that the spectrum of a single Cardy boundary $(r, s)$ is,

$$
\left\{\left(r^{\prime}, s^{\prime}\right)+\text { desc. : } 0<r^{\prime}<2 r, 0<s^{\prime}<2 s, r^{\prime}, s^{\prime} \text { odd }\right\}
$$

while the spectrum of boundary changing operators between $\left(r_{1}, s_{1}\right)$ and $\left(r_{2}, s_{2}\right)$ is

$$
\left\{\left(r^{\prime}, s^{\prime}\right)+\text { desc. : } \begin{array}{l}
\left|r_{1}-r_{2}\right|<r^{\prime}<r_{1}+r_{2}, r_{1}+r_{2}+r^{\prime} \text { odd, } \\
\left|s_{1}-s_{2}\right|<s^{\prime}<s_{1}+s_{2}, s_{1}+s_{2}+s^{\prime} \text { odd }
\end{array}\right\},
$$


where the weights are given by

$$
h_{r, s}=\frac{(r-s)^{2}}{4}+\frac{r^{2}-s^{2}}{4(m+1)}+\frac{r^{2}-1}{4(m+1)^{2}}+\ldots
$$

For a general boundary condition there are a finite number of primary fields and their weights concentrate around the integers and the integers plus $\frac{1}{4}$. From (2.11), the relevant fields with $h \leq 1$ fall into three sets: $\phi_{r, r}$ with $h \sim 0, \phi_{r, r+2}$ with $h \sim 1$ (these two sets can exist on a single boundary or as boundary changing operators) and $\phi_{r, r \pm 1}$ with $h \sim \frac{1}{4}$ (which only exist as boundary changing operators). As well as these, we will also be interested in the almost relevant fields $\phi_{r+2, r}$ with $h \sim 1$. We will see in section 3.1 that both $\phi_{r, r+2}$ and $\phi_{r+2, r}$ are important for perturbation theory in $1 /(m+1)$. Perturbations by the other fields must be studied by other methods, see for example 10.

\subsection{The results of Recknagel et. al.}

In 14], Recknagel, Roggenkamp and Schomerus (RRS) studied perturbations of a single Cardy boundary condition by the field $\phi_{13}$ in the $c \rightarrow 1, m \rightarrow \infty$ limit using perturbation theory in $1 /(m+1)$. After choosing a renormalisation scheme in which the boundary theory had a non-trivial perturbative fixed point, the authors evaluated the boundary entropy at this new fixed point. This they compared term by term in $1 /(m+1)$ with $(2.5)$ and so derived a set of integer equations that allowed them to conjecture the end points of a generic $\phi_{13}$ flow. The result,

$$
(r, s) \rightarrow \oplus_{i=1}^{\min \{r, s\}}(r+s+1-2 i, 1)
$$

In this paper we will extend these studies to general $\phi_{r, r+2}$ perturbations and in particular to $\phi_{13}$ boundary changing operators. In fact we will find that the latter will have something to say about the former.

\section{Boundary Perturbations}

Here we study perturbations of a boundary conformal field theory on a disc of circumference $L$ with boundary condition $\omega=\oplus_{a=1}^{n} \omega_{a}$, a superposition of Cardy boundary conditions. The perturbed theory is defined by the prescription,

$$
\begin{gathered}
\left\langle\psi_{1} \ldots \psi_{n}\right\rangle_{\omega ; \lambda_{\phi}}=\left\langle P \psi_{1} \ldots \psi_{n} e^{\delta S}\right\rangle_{\omega} \\
\delta S=\int_{\partial M} d x\left[\sum_{\phi, a, b} \lambda_{\phi}^{a b} \varepsilon^{-y_{\phi}} \phi^{a b}(x)+\sum_{a} \alpha_{a} \varepsilon^{-1} \mathbb{1}_{a}(x)\right]
\end{gathered}
$$

where $\mathrm{P}$ denotes path ordering, the $\psi_{i}=\psi_{i}\left(z_{i}, \bar{z}_{i}\right)$ are renormalised fields, $y_{\phi}=1-h_{\phi}, h_{\phi}$ is the conformal weight of $\phi$ and we have introduced a factor of $\varepsilon^{-y_{\psi}}$ to make the couplings dimensionless. We have also separated the identity field of each component boundary $\omega_{a}$ (denoted by $\phi_{11}^{a a}=\mathbb{1}_{a}$ with coupling $\alpha_{a}$ ) from the general sum of perturbing fields. The identity fields will play a specific role in what is to follow so we identify them with a special 
notation. To preserve unitarity, the perturbing operator is chosen to be Hermitean thus for the boundary changing couplings we have $\lambda^{a b}=x+i y, \lambda^{b a}=x-i y$ for real variables $x$ and $y$. In general, (3.1) is UV divergent and must be regularised. We choose to restrict integrals of the non-identity fields to the region $\left|x_{i}-x_{j}\right|>\varepsilon$ where $\varepsilon$ acts as our regulator.

We will evaluate (3.1) in perturbation theory as a power series in $1 /(m+1)$ in a renormalisation scheme with non-trivial renormalisation group fixed points such that $\lambda=O(1 /(m+1))$. It turns out that such a scheme is defined by the requirement that

$$
\left.\lim _{\varepsilon \rightarrow 0} \varepsilon \partial_{\varepsilon} P e^{\delta S}\right|_{\lambda_{R}}=0
$$

with the renormalisation conditions

$$
\lambda_{\text {bare }}=\left.\lambda_{R}\right|_{\varepsilon=L},
$$

where $\lambda_{\text {bare }}$ is the bare coupling $\lambda$ of (3.1).

The first task is to calculate the $\beta$-functions in this scheme. To do this we use a trick advocated in [8], whereby we use the relation $\beta_{\lambda} \equiv L d_{L} \lambda_{R}=\left.\left(\left.\varepsilon \partial_{\varepsilon} \lambda_{\text {bare }}\right|_{\lambda_{R}}\right)\right|_{\lambda_{\text {bare }}=\lambda_{R}}$ (which follows from (3.3) and the fact that $\beta_{\lambda}$ is purely a function of $\lambda_{R}$ ) and consider (3.2) expanded as a power series in the couplings of the non-identity fields. The first term in this expansion contains only identity fields,

$$
0=P\left[e^{\sum_{a} \alpha_{a} \varepsilon^{-1} \int d x \mathbb{1}_{a}(x)} \sum_{a}\left(\varepsilon \partial_{\varepsilon} \alpha_{a}-\alpha_{a}\right) \varepsilon^{-1} \int d x \mathbb{1}_{a}(x)\right],
$$

which requires $\beta_{\alpha_{a}}=\alpha_{a}$. Using this result, the first order contribution to (3.2) becomes,

$$
0=\left(\varepsilon \partial_{\varepsilon} \lambda_{k}-y_{k} \lambda_{k}\right) \varepsilon^{-y_{k}} \int_{0}^{L} d x e^{\alpha_{a} \varepsilon^{-1} x \mathbb{1}_{a}} \phi_{k}^{a c}(x) e^{\alpha_{c} \varepsilon^{-1}(L-x) \mathbb{1}_{c}} .
$$

Hence $\beta_{\lambda_{k}}=y_{k} \lambda_{k}$. Moving second order we have,

$$
\begin{aligned}
\varepsilon \partial_{\varepsilon} & {\left[\lambda_{i} \lambda_{j} \varepsilon^{-y_{i}-y_{j}}\right.} \\
& \left.\times \int_{0}^{L} d x_{1} d x_{2} \theta\left(x_{2}-x_{1}-\varepsilon\right) e^{\alpha_{a} \varepsilon^{-1} x_{1} \mathbb{1}_{a}} \phi_{i}^{a b}\left(x_{1}\right) e^{\alpha_{b} \varepsilon^{-1}\left(x_{2}-x_{1}\right) \mathbb{1}_{b}} \phi_{j}^{b c}\left(x_{2}\right) e^{\alpha_{c} \varepsilon^{-1}\left(L-x_{2}\right) \mathbb{1}_{c}}\right] \\
= & -\lambda_{i} \lambda_{j} \varepsilon^{-y_{i}-y_{j}} \int_{0}^{L} d x e^{\alpha_{a} \varepsilon^{-1} x \mathbb{1}_{a}} \phi_{i}^{a b}(x) e^{\alpha_{b}} \phi_{j}^{b c}(x+\varepsilon) e^{\alpha_{c} \varepsilon^{-1}(L-x-\varepsilon) \mathbb{1}_{c}} \\
= & -\lambda_{i} \lambda_{j} \varepsilon^{-y_{i}-y_{j}} \\
& \times \int_{0}^{L} d x e^{\alpha_{a} \varepsilon^{-1}\left(x+\frac{1}{2} \varepsilon\right) \mathbb{1}_{a}} \sum_{k} C_{i j}^{(a b c) k} e^{\alpha_{b}-\frac{1}{2}\left(\alpha_{a}+\alpha_{c}\right)} \varepsilon^{y_{i}+y_{j}-y_{k}} \phi_{k}^{a c}\left(x+\frac{1}{2} \varepsilon\right) e^{\alpha_{c} \varepsilon^{-1}\left(L-\left(x+\frac{1}{2} \varepsilon\right)\right) \mathbb{1}_{c}} \\
= & -\lambda_{i} \lambda_{j} \sum_{k} \varepsilon^{-y_{k}} C_{i j}^{(a b c) k} e^{\alpha_{b}-\frac{1}{2}\left(\alpha_{a}+\alpha_{c}\right)} \int_{-\frac{1}{2} \varepsilon}^{L-\frac{1}{2} \varepsilon} d x e^{\alpha_{a} \varepsilon^{-1} x \mathbb{1}_{a}} \phi_{k}^{a c}(x) e^{\alpha_{c} \varepsilon^{-1}(L-x) \mathbb{1}_{c}}
\end{aligned}
$$

\footnotetext{
* The vertical bar in (3.2) denotes "evaluated at constant ...". Also $\partial_{\varepsilon}=\frac{\partial}{\partial \varepsilon}$ and later we will use $d_{L}=\frac{d}{d L}$.
} 
where $\theta(x)$ is the usual step function and we have used the lower order results $\varepsilon \partial_{\varepsilon}\left(\lambda \varepsilon^{-y}\right)=0$ and $\varepsilon \partial_{\varepsilon}\left(\alpha \varepsilon^{-1}\right)=0$. From (3.8) one can read off the second order correction to the $\beta$-functions,

$$
\begin{aligned}
& \beta_{\lambda_{i}^{a c}}=y_{i} \lambda_{i}^{a c}+\sum_{j, k} C_{j k}^{(a b c) i} \lambda_{j}^{a b} \lambda_{k}^{b c} e^{\alpha_{b}-\frac{1}{2}\left(\alpha_{a}+\alpha_{c}\right)}, \\
& \beta_{\alpha_{a}}=\alpha_{a}+\sum_{j, k} C_{j k}^{(a b a)} \mathbb{1} \lambda_{j}^{a b} \lambda_{k}^{b a} e^{\alpha_{b}-\alpha_{a}},
\end{aligned}
$$

where we have added the boundary indices to the coupling constants. We should also calculate the third order corrections to the identity field, however they only play a passive role in the calculations to follow so we do not write them explicitly.

\subsection{What can we study from (3.9) and (3.10)?}

We are interested in perturbation theory, hence for the formulae derived so far to be valid, the couplings must be smalli'. Let us assume all the couplings in the theory are of order $y \equiv \frac{2}{m+1}=y_{13}$ and look for fixed points in (3.9, 3.10). For our purposes, it is sufficient to consider the non-identity $\beta$-functions to $O\left(y^{2}\right)$ and the identity $\beta$-functions to $O\left(y^{3}\right)$. We call fields with $y_{i} \sim y$ (namely $\phi_{r, r+2}$ and $\phi_{r+2, r}$ ) "perturbative" while all other fields are "nonperturbative". Assuming the structure constants in (3.9, 3.10) are of $O(1)$ as $m \rightarrow \infty$, the fixed points of the non-perturbative fields are all $O\left(y^{2}\right)$. Consequently we see that to $O\left(y^{2}\right)$, the non-linear terms in the $\beta$-functions contain only perturbative fields and in particular, we can neglect the exponentials of identity couplings. Furthermore, the $O\left(y^{3}\right)$ corrections to the identity field $\beta$-functions also contain only perturbative fields. This is because conformal invariance of the two point function requires $C_{j k}^{(a b c) \mathbb{1}} \neq 0 \Longrightarrow y_{j}=y_{k}$. Finally we note that the fixed points of the system (3.9, 3.10) are determined entirely from the $\beta$-functions of the perturbative fields.

Which fields should we include in the sums of (3.9, 3.10)? Naively one would include only relevant fields, but this would be wrong. We are considering perturbation theory in $y=2 /(m+1)$ so any factors of $\varepsilon^{-y_{k}}$ for $y_{k} \sim \pm y$ found in the perturbative expansion should also be expanded $\varepsilon^{-y_{k}}=1-y_{k} \ln \varepsilon+\ldots$ and the logarithms subtracted order by order. This prescription introduces extra divergences due to the fusing of relevant fields to perturbative irrelevant fields. With this in mind, the sums of (3.9, 3.10) should include both the relevant and the perturbative irrelevant fields.

One can see this another way. We are expanding in $1 /(m+1)$ about the $c=1$ boundary theory. At $c=1$ all the perturbative fields are marginal and generate each other as counterterms and so must be included in the $\beta$-functions. The fact that some of these fields turn out to be irrelevant through higher order corrections is unimportant, they still have a non-trivial effect on the dynamics of the renormalisation group flow.

\footnotetext{
${ }^{\dagger}$ If we are careful we could allow the identity field couplings to be large since we have treated those non-perturbatively. However in the examples considered in this paper, the only fixed points that are both nonperturbative in the identity fields and perturbative in the non-identity fields involve setting all the boundary changing operators to zero. In this case the perturbation by identity fields acts trivially, projecting onto some subset of component boundaries
} 
We end this section by emphasizing that there are a finite number of fields in the $\beta$ functions (3.9, 3.10) and so one can use them to study perturbations by all perturbative fields. This is in contrast to the bulk studies of Zamolodchikov [17] where as $1 /(m+1)$ gets small, the number of relevant bulk fields gets very large and in all but a few cases, the perturbation theory becomes unwieldy.

\subsection{Two Examples, Finding the Fixed Points}

\subsubsection{Example 1 : Boundary Changing Perturbations}

As our first example, we will consider $\phi_{13}$ perturbations on the boundary condition $\omega=$ $\oplus_{a=1}^{n} \omega_{a}=\oplus_{a=1}^{n}(r, s+2 a-2)$. The spectrum of $\phi_{13}$ fields consists of,

- Boundary changing operators $\phi_{13}^{a, a+1}=\psi_{a}$ acting between the boundaries $\omega_{a}$ and $\omega_{a+1}$, we denote their coupling by $\mu_{a}$.

- Their conjugate fields, $\phi_{13}^{a+1, a}=\psi_{a}^{\dagger}$, whose coupling we denote by $\mu_{a}^{\dagger}$.

- An ordinary $\phi_{13}^{a, a}=\phi_{a}$ field on each boundary with coupling $\lambda_{a}$.

Couplings to all other fields (except the identity fields) can be consistently set to zero. Following the discussion of section 3.1, to find the fixed points we need only consider the $\beta$-functions of the perturbative fields,

$$
\begin{aligned}
& \beta_{\lambda_{a}}=y \lambda_{a}+C_{. \cdot}^{(a a a) \cdot} \lambda_{a}^{2}+C_{. \cdot}^{(a b a) \cdot} \mu_{a} \mu_{a}^{\dagger}+C_{. \cdot}^{(a c a) \cdot} \mu_{c} \mu_{c}^{\dagger}, \\
& \beta_{\mu_{a}}=\left(y+C_{\cdot \cdot}^{(a a b) \cdot} \lambda_{a}+C_{\cdot .}^{(a b b) \cdot} \lambda_{b}\right) \mu_{a}, \\
& \beta_{\mu_{a}^{\dagger}}=\left(y+C_{. \cdot}^{(b a a) \cdot} \lambda_{a}+C_{\cdot .}^{(b b a) \cdot} \lambda_{b}\right) \mu_{a}^{\dagger},
\end{aligned}
$$

where the a dot denotes $\phi_{13}, b=a+1$ and $c=a-1$, it is also understood that $\lambda_{0}=\mu_{0}=$ $\mu_{0}^{\dagger}=\lambda_{n+1}=\mu_{n}=\mu_{n}^{\dagger}=0$. When $s=1$ we also need to account for the fact that there is no $\phi_{13}$ field on the $(1,1)$ boundary.

For the A Series minimal models $C_{. .}^{(b a a) .}=C_{. .}^{(a a b)}$. and $C_{. .}^{(b b a) .}=C_{. .}^{(a b b) \text {. }}$ so we combine (3.12) and (3.13) into a single equation for $\hat{\mu}=\left(\mu_{a} \mu_{a}^{\dagger}\right)^{\frac{1}{2}}$,

$$
\beta_{\hat{\mu}_{a}}=\left(y+C_{. .}^{(a a b) \cdot} \lambda_{a}+C_{. .}^{(a b b) \cdot} \lambda_{b}\right) \hat{\mu}_{a} .
$$

We now wish to study the fixed points of (3.11,3.14). Without loss of generality we can assume all the couplings to boundary changing operators are non-zero (for if one were zero, between $\omega_{b}$ and $\omega_{b+1}$ say, then the equations decompose into two independent sets, $\oplus_{a=1}^{b} \omega_{a}$ and $\left.\oplus_{a=b+1}^{n} \omega_{a}\right)$. Hence $\beta_{\hat{\mu}_{a}}=0$ implies,

$$
0=y+C_{. .}^{(a a b) \cdot} \lambda_{a}+C_{. .}^{(a b b) \cdot} \lambda_{b}, \quad b=a+1, \quad a=1, \ldots, n-1,
$$

and we can find all the $\lambda_{a}$ in terms of a particular lambda, $\lambda$ say. Substituting these expressions into (3.11) for $a=1$ gives $\hat{\mu}_{1}$ in terms of $\lambda$, then by iteration we find all $\hat{\mu}$ using (3.11) for $a=2, \ldots, n-1$. Finally we are left with (3.11) for $\lambda_{n}$, this is a quadratic in $\lambda$ which has two solutions. The structure constants are known from [15] and those relevant to 
this calculation are collected in appendix A.1. Inserting them, we find that the boundary changing couplings are the same for each solution,

$$
\mu_{a}^{*} \mu_{a}^{\dagger}=\frac{y^{2}}{4} \frac{(s+n+a-1)(s+a-1)(n-a) a}{(s+2 a-1)(s+2 a-2)}, \quad a=1, \ldots, n-1,
$$

while the ordinary fields are given by,

$$
\begin{aligned}
& \lambda_{a}^{*(1)}=-\frac{y}{2 \sqrt{2}} \frac{2 a^{2}-4 a+2 a s-3 s+s^{2}+n s-n+2}{\sqrt{s+2 a-3} \sqrt{s+2 a-1}}, \quad a=1, \ldots, n, \\
& \lambda_{a}^{*(2)}=-\frac{y}{2 \sqrt{2}} \frac{2 a^{2}-4 a+2 a s-s-s n+n+1}{\sqrt{s+2 a-3} \sqrt{s+2 a-1}}, \quad a=1, \ldots, n .
\end{aligned}
$$

These solutions are also valid in the case $s=1$, in which case the two solutions $\lambda^{*(1)}$ and $\lambda^{*(2)}$ become identified and $\lambda_{1}^{*}$ vanishes because there is no $\phi_{13}$ field on the $(1,1)$ boundary.

A point to note is that we were only able to solve for $\hat{\mu}_{a}=\mu_{a} \mu_{a}^{\dagger}$ which is invariant under $\mu_{a} \rightarrow e^{i \theta} \mu_{a}, \mu_{a}^{\dagger} \rightarrow e^{-i \theta} \mu_{a}^{\dagger}$. This means that we have in fact found a continuum of solutions with dimension equal to the number of non-zero boundary changing operators, i.e. the fixed points contain marginal deformations. Although we expect this marginality will be lifted at the next order in perturbation theory (there are no marginal boundary operators in any minimal model), we will take a moment to study the moduli more carefully. In a perturbation involving only one pair of boundary changing operators between boundaries $a$ and $b$, consider the small change $\mu \rightarrow \mu+i \theta \mu, \mu^{\dagger} \rightarrow \mu^{\dagger}-i \theta \mu^{\dagger}$. At the fixed point, this corresponds to adding the perturbation,

$$
\delta S=i \theta \varepsilon^{-y} \int_{\partial M} d x\left[\mu \psi-\mu^{\dagger} \psi^{\dagger}\right]
$$

So we are interested in the nature of the operator $\mu \psi-\mu^{\dagger} \psi^{\dagger}$ at the fixed point. To this end we consider the following object at the fixed point,

$$
\frac{\partial}{\partial x}\left\langle\left[\mathbb{1}_{a}(x)-\mathbb{1}_{b}(x)\right] X\right\rangle_{\lambda^{*}, \mu^{*}},
$$

where $X$ denotes the insertions away from $x$. On a small neighbourhood $\left(x_{0}, x_{1}\right)$ of $x$, away from the insertions, the identity field is given by the perturbative expansion,

$$
\left.\mathbb{1}_{a}(x)\right|_{\lambda^{*}, \mu^{*}}=\mathbb{1}_{a}(x)+\lambda_{a} \varepsilon^{-y} \int_{x_{0}}^{x_{1}} d y \phi_{a}(y)+\mu \varepsilon^{-y} \int_{x}^{x_{1}} d y \psi(y)+\mu^{\dagger} \varepsilon^{-y} \int_{x_{0}}^{x} d y \psi^{\dagger}(y)+O\left(\lambda^{2}\right) .
$$

Then using $\partial_{x} \mathbb{1}_{a}(x)=0$ we find,

$$
\left.\frac{\partial}{\partial x}\left(\mathbb{1}_{a}(x)-\mathbb{1}_{b}(x)\right)\right|_{\lambda^{*}, \mu^{*}} \sim-2 \varepsilon^{-y}\left(\mu \psi(x)-\mu^{\dagger} \psi^{\dagger}(x)\right),
$$

indicating the marginal perturbations are generated by derivative fields; the level one descendent of a $h \sim 0$ field. This is as we would expect, the only marginal fields to this order are the level one descendants of $\phi_{r r}$ fields. One does not expect perturbations by derivative fields to have any effect on the theory, in the language of [6] they are redundant fields, and so 
one may think of these "lines of marginal stability" as coordinate singularities in the space of boundary field theories. Even so, they are useful in identifying which boundary theory is represented at the fixed point, we will discuss this further in section 4

We end the section with a summary of the fixed points we have found with the example of $\omega=(1,1) \oplus(1,3) \oplus(1,5)$. The fixed points, $\left\{\lambda_{2}, \lambda_{3}, \mu_{1} \mu_{1}^{\dagger}, \mu_{2} \mu_{2}^{\dagger}\right\}$, for this system are,

- The origin $\{0,0,0,0\}$.

- 2 points studied by RRS $\{-y, 0,0,0\},\{0,-\sqrt{3} y, 0,0\}$ together with the combination $\{-y,-\sqrt{3} y, 0,0\}$.

- 1 boundary changing fixed point $\left\{-\frac{1}{2} y,-\frac{\sqrt{3}}{2} y, y^{2}, \frac{5}{12} y^{2}\right\}$.

- 3 boundary changing fixed points involving just two boundaries, $\left\{-\frac{1}{2} y, 0, \frac{3}{8} y^{2}, 0\right\}$, $\left\{-\frac{5}{4} y,-\frac{3 \sqrt{3}}{4} y, 0, \frac{5}{16} y^{2}\right\},\left\{\frac{1}{4} y,-\frac{\sqrt{3}}{4} y, 0, \frac{5}{16} y^{2}\right\}$.

- Finally, the $(1,1) \oplus(1,3)$ boundary changing fixed point with a RRS flow on the remaining $(1,5)$ boundary, $\left\{-\frac{1}{2} y,-\sqrt{3} y, \frac{3}{8} y^{2}, 0\right\}$.

All in all, 9 fixed points. The general picture is clear. Our task now is to identify which boundary condition is present at each fixed point. This will be the subject of sections 4 and 5.

\subsubsection{Example 2: $\phi_{35}$ Perturbations}

Our second example will be perturbation theory on the boundary condition $\omega=(2, p)$. From the discussion in section 3.1 we only need to consider the $\beta$-functions of the perturbative fields: $\left\{\phi=\phi_{13}, \tilde{\phi}=\phi_{31}, d_{3}=\frac{m+1}{2} L_{-1} \phi_{33}, \psi=\phi_{35}\right\}$ with couplings $\left\{\lambda_{\phi}, \lambda_{\tilde{\phi}}, \lambda_{d}, \lambda_{\psi}\right\}$ respectively.

The normalisation of the derivative field follows from the choice $C_{d d}^{(. .)} \mathbb{1}=1$. Also unitarity requires that $\lambda_{d}$ be purely imaginary. Because the descendent field is redundant, we may consistently set its coupling to zero. However, we choose not to so that parallels with the boundary changing case are easier to see.

The system has the $\beta$-functions,

$$
\begin{aligned}
& \beta_{\phi}=y \lambda_{\phi}+C_{\phi \phi}^{(. .) \phi} \lambda_{\phi}^{2}+C_{d d}^{(. .) \phi} \lambda_{d}^{2}+C_{\psi \psi}^{(. .) \phi} \lambda_{\psi}^{2}, \\
& \beta_{\tilde{\phi}}=-y \lambda_{\tilde{\phi}}+C_{\tilde{\phi} \tilde{\phi}}^{(\ldots) \tilde{\phi}} \lambda_{\tilde{\phi}}^{2}+C_{d d}^{(. .) \dot{\phi}} \lambda_{d}^{2}+C_{\psi \psi}^{(. .) \tilde{\phi}} \lambda_{\psi}^{2}, \\
& \beta_{d}=2 C_{\phi d}^{(. .) d} \lambda_{\phi} \lambda_{d}+2 C_{\tilde{\phi} d}^{(. .) d} \lambda_{\tilde{\phi}} \lambda_{d}+2 C_{\psi d}^{(. .) d} \lambda_{\psi} \lambda_{d}, \\
& \beta_{\psi}=2 y \lambda_{\psi}+C_{\psi \psi}^{(. .) \psi} \lambda_{\psi}^{2}+C_{d d}^{(. .) \psi} \lambda_{d}^{2}+2 C_{\phi \psi}^{(. .) \psi} \lambda_{\phi} \lambda_{\psi}+2 C_{\tilde{\phi} \psi}^{(. .) \psi} \lambda_{\tilde{\phi}} \lambda_{\psi},
\end{aligned}
$$

where in this case the dots denote the $(2, p)$ boundary. The structure constants are collected in appendix A.2. Inserting them into (3.23 3.26) we obtain the following four isolated fixed 
points,

$$
\left\{\lambda_{\phi}, \lambda_{\tilde{\phi}}, \lambda_{d}, \lambda_{\psi}\right\}=\left\{\begin{array}{l}
f_{1}=\left\{0, \frac{1}{2} \sqrt{\frac{3}{2}} y, 0,0\right\} \\
f_{2}=\left\{-\frac{(p-2) \sqrt{p^{2}-1}}{4 \sqrt{2} p} y, \frac{(p+1)(p+2)}{4 \sqrt{6} p} y, 0, \frac{\sqrt{\left(p^{2}-4\right)\left(p^{2}-1\right)}}{4 \sqrt{3} p} y\right\}, \\
f_{3}=\left\{-\frac{(p+2) \sqrt{p^{2}-1}}{4 \sqrt{2} p} y,-\frac{(p-1)(p-2)}{4 \sqrt{6} p} y, 0,-\frac{\sqrt{\left(p^{2}-4\right)\left(p^{2}-1\right)}}{4 \sqrt{3} p} y\right\} \\
f_{4}=\left\{-\frac{\sqrt{p^{2}-1}}{2 \sqrt{2}} y, 0,0,0\right\},
\end{array}\right.
$$

together with two continua due to the derivative perturbation which we parameterise by $\theta \in[0,2 \pi)$, with $A(\theta)=1-\cos \theta$,

$$
\begin{aligned}
& \left\{\lambda_{\phi}, \lambda_{\tilde{\phi}}, \lambda_{d}, \lambda_{\psi}\right\}= \\
& \left\{\begin{array}{l}
f_{5}=\left\{-A \frac{\sqrt{p^{2}-1}}{\sqrt{2} p^{2}} y, A \frac{\left(p^{2}-1\right)}{\sqrt{6} p^{2}} y, i \sin \theta \frac{\sqrt{p^{2}-1}}{2 p} y,-A \frac{\sqrt{\left(p^{2}-1\right)\left(p^{2}-4\right)}}{2 \sqrt{3} p^{2}} y\right\}, \\
f_{6}=\left\{-\left(p^{2}-2 A\right) \frac{\sqrt{p^{2}-1}}{2 \sqrt{2} p^{2}} y, \sqrt{\frac{3}{8}} y-A \frac{\left(p^{2}-1\right)}{\sqrt{6} p^{2}} y, i \sin \theta \frac{\sqrt{p^{2}-1}}{2 p} y, A \frac{\sqrt{\left(p^{2}-1\right)\left(p^{2}-4\right)}}{2 \sqrt{3} p^{2}} y\right\} .
\end{array}\right.
\end{aligned}
$$

The origin is contained as $f_{5}(\theta=0)$.

\section{A Study of the $\beta$-functions}

Having found the fixed points, we would now like to identify the associated conformal boundary theories and discover which points are connected to which by relevant flows. The fact that the first order term in the $\beta$-function is universal provides a way of studying the nature of a fixed point directly from the $\beta$-functions. Let $\lambda^{*}$ be a non-trivial fixed point for the generic set of equations,

$$
\beta_{\lambda_{i}}=y_{i} \lambda_{i}+\sum_{j, k} C_{j k}{ }^{i} \lambda_{j} \lambda_{k}
$$

A couple of remarks: (i) We imagine (4.30) to contain the $\beta$-functions for all fields - both relevant and irrelevant. (ii) The constants $C_{j k}{ }^{i}$ depend on the choice of renormalisation scheme and will in general differ from those in the OPE.

Re-expressing (4.30) about the new fixed point, $\lambda \rightarrow \lambda^{*}+\delta \lambda$, we find

$$
\beta_{\delta \lambda_{i}}=\left.\sum_{j} \frac{\partial \beta_{\lambda_{i}}}{\partial \lambda_{j}}\right|_{\lambda^{*}} \delta \lambda_{j}+O\left(\delta \lambda^{2}\right) .
$$

Now by diagonalising the first term, we return the $\beta$-function to the generic form (4.30) and can read off the spectrum of fields.

Looking for fixed points in (4.30) we know only the perturbative fields have $\lambda_{a}^{*} \sim y$ while all other fields have $\lambda_{n}^{*} \sim y^{2}$, so to first order in $y$,

$$
\left.B_{i j}^{*} \equiv \frac{\partial \beta_{\lambda_{i}}}{\partial \lambda_{j}}\right|_{\lambda^{*}}=y_{i} \delta_{i j}+\sum_{a}\left(C_{j a}^{i}+C_{a j}{ }^{i}\right) \lambda_{a}^{*}
$$


An application of Rayleigh-Schrodinger, gives the change in $y_{n}$ for the non-perturbative fields along a flow,

$$
\delta y_{n}=\sum_{a}\left(C_{n a}^{n}+C_{a n}^{n}\right) \lambda_{a}^{*}
$$

More interesting are the perturbative fields with $y_{i} \sim 0$. For example, to the order we are considering,

$$
\operatorname{dim} \operatorname{ker} B_{a, b}^{* U V}-\operatorname{dim} \operatorname{ker} B_{a, b}^{* I R}=\text { The change in the number of Cardy boundaries . }
$$

To see this note that $\operatorname{dim} \operatorname{ker} B_{a, b}^{*}$ counts the number of fields with $y_{i}=0$. Such fields are the level one descendants of the fields $\phi_{r r}, r \neq 1$. From (4.33) and (2.11) we see that the total number of $\phi_{r r}$ fields (including the identity) remains constant between perturbative fixed points so any increase in the number of $y_{i}=0$ fields must correspond to a decrease in the number of $\phi_{11}$ fields. The number of $\phi_{11}$ fields on a boundary condition represents the number of Cardy boundary conditions in the superposition giving (4.34).

\subsection{Example 1 : Boundary Changing Perturbations}

We now apply this theory to the fixed points found in section 3.2.1. There it was noted that fixed points involving boundary changing operators actually contain lines of marginal stability. In the present discussion this corresponds to zero eigenvalues of $B_{i, j}^{*}$, one for each pair of non-zero boundary changing operators. This observation allows us to deduce the nature of the fixed points in the special case of $\omega=\oplus_{a=1}^{n}(1, s+2 a-2)$, for which the only perturbative fields are $\phi_{13}$. In this instance, each conjugate pair of non-zero boundary changing couplings contributes a zero eigenvalue to $B_{i, j}^{*}$, so increases the difference between the number of boundaries in the starting and ending superpositions by one. If all the boundary changing operators are non-zero then the endpoint will be a single boundary, and its spectrum must contain $n \phi_{r r}$ fields. This requires the end point boundary to be either $(n, p),(p, n)$, $(m-n, p)$ or $(m-p, n)$ for some $p \geq n$. To find $p$ and deduce which of the four options is taken we need some more information. This is provided by the boundary entropy and the one point functions of bulk fields as will be calculated in section 5 . For now we quote the zeroth order constraints $\mathrm{f}$. In the case of the boundary entropy, we have that in a perturbative flow from $\omega=\oplus_{a=1}^{n}\left(r_{a}, s_{a}\right)$ to $\omega^{\prime}=\left(r^{\prime}, s^{\prime}\right)$ or $\omega^{\prime}=\left(m-r^{\prime}, s^{\prime}\right)$ satisfies,

$$
\sum_{a=1}^{n} r_{a} s_{a}=r_{a}^{\prime} s_{a}^{\prime} .
$$

This implies the $p=s+n-1$. To narrow things down further we consider the zeroth order constraint coming from the bulk one point functions (2.7), allowing us to discount $(m-s-n+1, n)$ and $(m-n, s+n-1)$. Finally, we diagonalise $B_{a, b}^{*}$ and quote the results in the following table. From the spectrum we predict the endpoint.

\footnotetext{
${ }^{\ddagger}$ The zeroth order constraints follow simply from the fact we are doing perturbation theory in $1 /(m+1)$ and do not require any serious computation.
} 


\begin{tabular}{lllll}
\hline $\mathrm{n}$ & Spectrum of $\lambda^{*(1)}$ & Endpoint & Spectrum of $\lambda^{*(2)}$ & Endpoint \\
\hline 2 & $0, \pm y,-2 y$ & $(s+1,2)$ & $0, \pm y, 2 y$ & $(2, s+1)$ \\
3 & $0,0, \pm y, \pm 2 y,-3 y$ & $(s+2,3)$ & $0,0, \pm y, \pm 2 y, 3 y$ & $(3, s+2)$ \\
4 & $0,0,0, \pm y, \pm 2 y, \pm 3 y,-4 y$ & $(s+3,4)$ & $0,0,0, \pm y, \pm 2 y, \pm 3 y, 4 y$ & $(4, s+3)$ \\
\hline
\end{tabular}

Table 1 : The Identification of the fixed points of the $\oplus_{a=1}^{n}(1, s+2 a-2)$ systems.

The general pattern is clear and will be shown when we calculate the boundary entropy in section 5. For now we notice that any Cardy boundary condition can be constructed as flow from a superposition of $(1, s)$ boundary conditions. Thus there is a hope that we can study the perturbations of a Cardy boundary by looking at the $\beta$-functions of the superposition. To this end consider $\omega=(1, p-1) \oplus(1, p+1)$ which contains a boundary changing flow to $(2, p)$. The fixed points of flows starting from $\omega$ (by diagonalising as above) can be identified as follows,

\begin{tabular}{|c|c|c|c|c|c|c|}
\hline & Fixed Point & $\left\{\lambda_{1}, \lambda_{2}, \mu \mu^{\dagger}\right\}$ & & & Spectrum & Endpoint \\
\hline\{ & 0 & 0 & 0 & \} & $y, y, y, y$ & $(1, p-1) \oplus(1, p+1)$ \\
\hline\{ & $-\frac{\sqrt{p(p-2)}}{2 \sqrt{2}} y$ & 0 & 0 & \} & $-y, y, \frac{1}{2} p y, \frac{1}{2} p y$ & $(p-1,1) \oplus(1, p+1)$ \\
\hline\{ & 0 & $-\frac{\sqrt{p(p+2)}}{2 \sqrt{2}} y$ & 0 & \} & $-\frac{1}{2} p y,-\frac{1}{2} p y,-y, y$ & $(1, p-1) \oplus(p+1,1)$ \\
\hline & $-\frac{\sqrt{p(p-2)}}{2 \sqrt{2}} y$ & $-\frac{\sqrt{p(p+2)}}{2 \sqrt{2}} y$ & 0 & \} & $-y,-y,-y,-y$ & $(p-1,1) \oplus(p+1,1)$ \\
\hline\{ & $\frac{\sqrt{p-2}}{2 \sqrt{2 p}} y$ & $-\frac{\sqrt{p+2}}{2 \sqrt{2 p}} y$ & $\frac{p+1}{4 p} y^{2}$ & \} & $-y, 0, y, 2 y$ & $(2, p)$ \\
\hline\{ & $-\frac{(p+1) \sqrt{p-2}}{2 \sqrt{2 p}} y$ & $-\frac{(p-1) \sqrt{p+2}}{2 \sqrt{2 p}} y$, & $\frac{p+1}{4 p} y^{2}$ & \} & $-2 y,-y, 0, y$ & $(p, 2)$ \\
\hline
\end{tabular}

Table $\mathrm{g}$ : The Identification of the fixed points of the $(1, p-1) \oplus(1, p+1)$ system.

It is important to connect the fixed points with relevant flows, i.e. show that the fixed points which we have identified can actually be reached by a relevant perturbation. For the case of $\phi_{13}$ boundary changing perturbations on the boundary condition $\oplus_{a=1}^{n}(r, s+2 a-2)$, the $\beta$-functions (3.11 3.13) have the form,

$$
\beta_{i}=y \lambda_{i}+\sum_{j, k} C_{j k}{ }^{i} \lambda_{j} \lambda_{k}
$$

for some constant $y$. Consider a fixed point $\lambda^{*}$, then $\lambda_{i}(L)=\lambda_{i}^{*} x(L)$ is a solution to 4.36 if,

$$
\beta_{i}=\lambda_{i}^{*} L d_{L} x=y \lambda_{i}^{*} x+\sum_{j, k} C_{j k}^{i} \lambda_{j}^{*} \lambda_{k}^{*} x^{2}=y \lambda_{i}^{*} x-y \lambda_{i}^{*} x^{2},
$$

which is true if,

$$
L d_{L} x=y x(1-x) .
$$

By solving (4.38), it is easy to show that there is a solution flowing from the origin to any nontrivial fixed point of (3.11-3.13). Furthermore, we see from appendix A.1 that the structure 
constants, and hence the dynamics, are independent of $r$. We use this freedom to set $r=1$, then among the fixed points of $(3.11-3.13)$ is the totally stable point $\oplus_{a=1}^{n}(s+2 a-2,1)$ which corresponds to the end of a RRS flow on each component boundary. Re-diagonalising (3.11. 3.13) at this point we find it to again have the form (4.36) but with $y \rightarrow-y$. The argument above then shows that every fixed point of the $\phi_{13}$ system on $\oplus_{a=1}^{n}(r, s+2 a-2)$ contains a flow to some stable superposition of $(a, 1)$ boundary conditions possibly with multiplicities. These multiplicities indicate the appearance of Chan-Paton indices [13].

\subsection{Example $2: \phi_{35}$ Perturbations}

Using the fixed points found in section 3.2.2, we diagonalise the $\beta$-functions of the $(2, p)$ boundary and tabulate the result in table 3 .

\begin{tabular}{cll}
\hline Fixed Point & Spectrum & Endpoint \\
\hline$f_{1}$ & $\{y, y, y, y\}$ & $(1, p-1) \oplus(1, p+1)$ \\
$f_{2}$ & $\left\{-y, y, \frac{1}{2} p y, \frac{1}{2} p y\right\}$ & $(p-1,1) \oplus(1, p+1)$ \\
$f_{3}$ & $\left\{-\frac{1}{2} p y,-\frac{1}{2} p y,-y, y\right\}$ & $(1, p-1) \oplus(p+1,1)$ \\
$f_{4}$ & $\{-y,-y,-y,-y\}$ & $(p-1,1) \oplus(p+1,1)$ \\
$f_{5}$ & $\{-y, 0, y, 2 y\}$ & $(2, p)$ \\
$f_{6}$ & $\{-2 y,-y, 0, y\}$ & $(p, 2)$ \\
\hline
\end{tabular}

Table $\mathrm{O}$ : The Identification of the fixed points of the $(2, p)$ system.

In agreement with table 2. In fact it turns out that the $\beta$-functions for the $(2, p)$ system $3.23-3.26)$ are equal, by a linear redefinition of the couplings, to the $\beta$-functions of the $(1, p-1) \oplus(1, p+1)$ system 3.113 .13$)$ : First translate to the appropriate fixed point, diagonalise and rescale.

We are also interested as to which of these fixed points are connected by relevant flows. We have already seen that the $(1, p-1) \oplus(1, p+1)$ boundary flows to all the others, and that all the fixed points flow to $(p-1,1) \oplus(p+1,1)$. To discover which fixed points can be reached from the $(2, p)$ point, we integrate the $\beta$-functions numerically. In figure 11 we have plotted a series of flows emanating from the relevant directions of $f_{5}=(2,3)$ (similar pictures exist for other $p$ ). It is hoped the reader will get a feel for how the "out" surface of the $(2, p)$ boundary is embedded in the 3-dimensional space of couplings and believe that this surface does indeed contain non-trivial fixed points. Plotted in figure 2 is the projection of these flows onto the $\lambda_{\phi}, \lambda_{\psi}$ plane. 


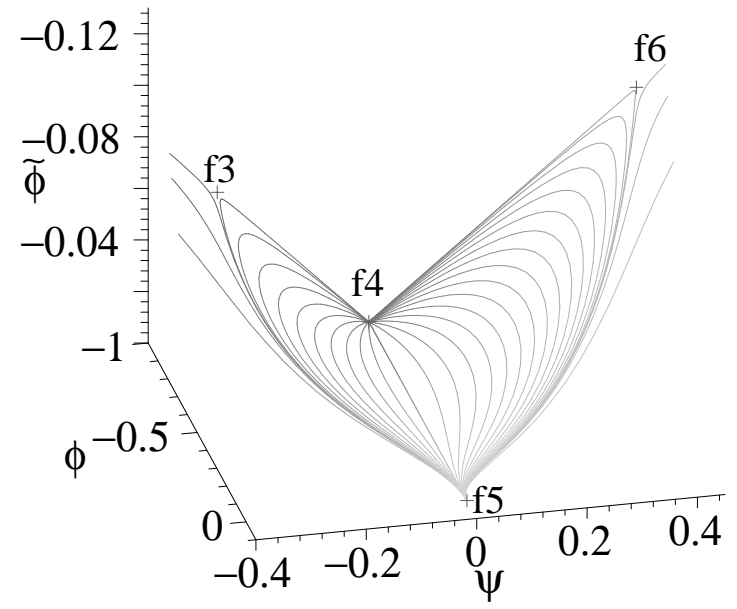

figure 1 : A three dimensional plot

of a series of flows emanating

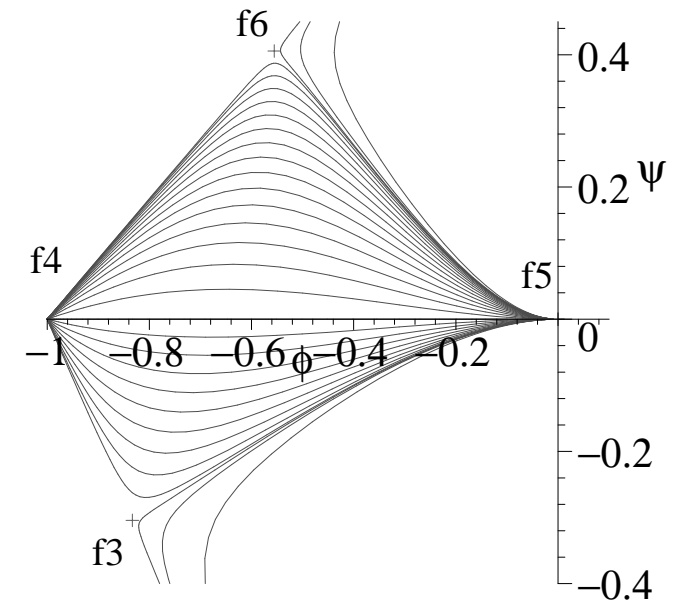

figure 0 : The projection of figure 1 onto the $\phi, \psi$ plane.

from the $f_{5}=(2,3)$ fixed point.

All in all, we obtain the following picture of flows,

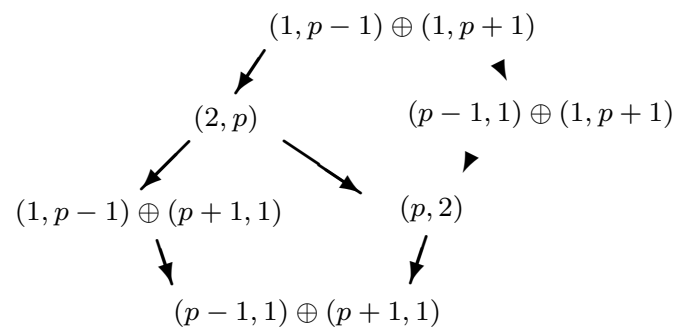

where it is true that a flow from $a \rightarrow b$ and from $b \rightarrow c$ implies that there exists a flow from $a \rightarrow c$. Sadly, numerical studies do not constitute proof that these fixed points can actually be reached.

\section{Deducing the Nature of the Fixed Points from the Boundary Entropy}

In this section we determine the nature of the fixed points of the boundary changing flows using the boundary entropy following the method of [14]. The idea is simply to calculate the boundary entropy $g$ in perturbation theory and compare it to the theoretical value (2.5) term by term in $1 /(m+1)$. This gives a set of integer equations which one can then solve.

We consider perturbations of the boundary condition $\omega=\oplus_{a=1}^{n} \omega_{a}=\oplus_{a=1}^{n}(r, s+2 a-2)$ by $\phi_{13}$ fields,

$$
\delta S=\int_{\partial M} d x\left[\sum_{a=1}^{n}\left(\lambda_{a} \varepsilon^{-y} \phi_{a}(x)+\alpha_{a} \varepsilon^{-1} \mathbb{1}_{a}(x)\right)+\sum_{a=1}^{n-1}\left(\mu_{a} \varepsilon^{-y} \psi_{a}(x)+\mu_{a}^{\dagger} \varepsilon^{-y} \psi_{a}^{\dagger}(x)\right)\right],
$$


with the notation introduced in section 3.2.1. The quantity of interest is the boundary entropy,

$$
\begin{aligned}
& g(\lambda, \mu, \alpha)= \sum_{a=1}^{n} e^{\alpha_{a} L \varepsilon^{-1}}\left\langle\mathbb{1}_{a}\right\rangle+\frac{1}{2} \varepsilon^{-2 y} \sum_{a=1}^{n} \lambda_{a}^{2} e^{\alpha_{a} L \varepsilon^{-1}} \int d x_{1} d x_{2}\left\langle P \phi_{a}\left(x_{1}\right) \phi_{i}\left(x_{2}\right)\right\rangle \\
&+\varepsilon^{-2 y} \sum_{a=1}^{n-1} \mu_{a} \mu_{a}^{\dagger} \int d x_{1} d x_{2}\left\langle P \psi_{a}\left(x_{1}\right) \psi_{a}^{\dagger}\left(x_{2}\right) e^{\varepsilon^{-1} \int d x\left(\alpha_{a} \mathbb{1}_{a}(x)+\alpha_{a+1} \mathbb{1}_{a+1}(x)\right)}\right\rangle \\
&+\frac{1}{6} \varepsilon^{-3 y} \sum_{a=1}^{n} \lambda_{a}^{3} e^{\alpha_{a} L \varepsilon^{-1}} \int d x_{1} d x_{2} d x_{3}\left\langle P \phi_{a}\left(x_{1}\right) \phi_{a}\left(x_{2}\right) \phi_{a}\left(x_{3}\right)\right\rangle \\
&+\varepsilon^{-3 y} \sum_{a=1}^{n-1} \sum_{b=a, a+1} \lambda_{b} \mu_{a} \mu_{a}^{\dagger} \int d x_{1} d x_{2} d x_{3}\left\langle P \phi_{b}\left(x_{1}\right) \psi_{a}\left(x_{2}\right) \psi_{a}^{\dagger}\left(x_{3}\right) e^{\varepsilon^{-1} \int d x\left(\alpha_{a} \mathbb{1}_{a}(x)+\alpha_{a+1} \mathbb{1}_{a+1}(x)\right)}\right\rangle, \\
&=g_{0}(\alpha)+g_{2}(\lambda, \alpha)+g_{2}(\mu, \alpha)+g_{3}(\lambda, \alpha)+g_{3}(\mu, \lambda, \alpha) .
\end{aligned}
$$

where we have pulled the identity fields out of the correlation functions where we can. The divergent integrals are regularised as discussed in section 3 .

Before continuing, we take a moment to consider the identity fields in the calculation to follow. To third order, the $\beta$-functions for the identity fields (3.10) do not contain higher order identity field corrections. Because of this it is useful to make a change of renormalisation scheme to remove the higher order corrections in the other fields as well, leaving one with a linear $\beta$-function,

$$
\beta_{\alpha^{\prime}}=\alpha^{\prime}
$$

To second order, this change is achieved by the transformation

$$
\alpha^{\prime}=\alpha+(1-2 y)^{-1} \sum_{j, k} C_{j k} \mathbb{1}_{j} \lambda_{k}
$$

which is non-singular and geometrically corresponds to projecting the flows onto the $\alpha^{\prime}$ plane. Under such a transformation, the renormalisation condition also changes,

$$
\left.\alpha_{R}\right|_{\varepsilon=L}=\left.\alpha_{\text {bare }} \longrightarrow \alpha_{R}^{\prime}\right|_{\varepsilon=L}=\alpha_{\text {bare }}+(1-2 y)^{-1} \sum_{j, k} C_{j k} \mathbb{1}_{\lambda_{j, b a r e}} \lambda_{k, \text { bare }} .
$$

To see the effect of the new condition, we solve $\beta_{\alpha^{\prime}}=L d_{L} \alpha^{\prime}=\alpha^{\prime}$,

$$
\frac{L}{\varepsilon} \alpha_{\text {bare }}=\alpha_{R}^{\prime}-\frac{L}{\varepsilon}(1-2 y)^{-1} \sum_{j, k} C_{j k} \mathbb{1}_{j, \text { bare }} \lambda_{k, \text { bare }} .
$$

The final term is generally interpreted as the ground state energy correction. That is to say, the scheme with a linear $\beta$-function for the identity fields is the scheme in which the identity fields are used to absorb the ground state energy corrections and only these corrections. Furthermore, at the fixed points $\alpha_{R}^{\prime}=0$. Because of this we choose simply to ignore both

\footnotetext{
${ }^{\S}$ Here we have used the fact that all the relevant fields have the same weight $y$; the generalisation is straightforward as is the inclusion of third order terms.
} 
the identity fields and the ground state energy corrections knowing that one is taking care of the other.

With that said, the calculation is almost identical to [2], from which we take the following results,

$$
\begin{aligned}
& g_{2}\left(\lambda_{a}\right)=\frac{1}{2} \varepsilon^{-2 y} \lambda_{a}^{2} \int d x_{1} d x_{2}\left\langle P \phi_{a}\left(x_{1}\right) \phi_{a}\left(x_{2}\right)\right\rangle=-\lambda_{a}^{2} \pi^{2} y\left(\frac{\varepsilon}{L}\right)^{-2 y} C_{. .}^{(a a a)} \mathbb{1}_{1}\left\langle\mathbb{1}_{a}\right\rangle, \\
& g_{2}\left(\mu_{a}\right)=\varepsilon^{-2 y} \mu_{a} \mu_{a}^{\dagger} \int d x_{1} d x_{2}\left\langle P \psi_{a}\left(x_{1}\right) \psi_{a}^{\dagger}\left(x_{2}\right)\right\rangle=-\mu_{a} \mu_{a}^{\dagger} \pi^{2} y\left(\frac{\varepsilon}{L}\right)^{-2 y} C_{. .}^{(a b a)} \mathbb{1}\left\langle\mathbb{1}_{a}\right\rangle, \\
& g_{3}\left(\lambda_{a}\right)=\frac{1}{6} \varepsilon^{-3 y} \lambda_{a}^{3} \int d x_{1} d x_{2} d x_{3}\left\langle P \phi_{a}\left(x_{1}\right) \phi_{a}\left(x_{2}\right) \phi_{a}\left(x_{3}\right)\right\rangle \\
& =\lambda_{a}^{3} \pi^{2}\left(\frac{\varepsilon}{L}\right)^{-3 y}\left[-\frac{8}{3}+2\left(\frac{\varepsilon}{L}\right)^{y}\right] C_{. .}^{(a a a) \cdot} C_{. .}^{(a a a)} \mathbb{1}_{1}\left\langle\mathbb{1}_{a}\right\rangle, \\
& g_{3}\left(\lambda_{a}, \mu_{b}\right)=\varepsilon^{-3 y} \lambda_{a} \mu_{b} \mu_{b}^{\dagger} \int d x_{1} d x_{2} d x_{3}\left\langle P \phi_{a}\left(x_{1}\right) \psi_{b}\left(x_{2}\right) \psi_{b}^{\dagger}\left(x_{3}\right)\right\rangle \\
& =3 \lambda_{a} \mu_{b} \mu_{b}^{\dagger} \pi^{2}\left(\frac{\varepsilon}{L}\right)^{-3 y}\left[-\frac{8}{3}+2\left(\frac{\varepsilon}{L}\right)^{y}\right] C_{. .}^{(b a a)} \cdot C_{. .}^{(a b a)} \mathbb{1}_{\text {. }}\left\langle\mathbb{1}_{a}\right\rangle .
\end{aligned}
$$

We also solve the $\beta$-function equations (see appendix A.3) to express the bare couplings in terms of the renormalised ones,

$$
\begin{aligned}
& \left(\frac{\varepsilon}{L}\right)^{-y} \lambda_{a}=\lambda_{a}^{R}-\frac{1}{y}\left(1-\left(\frac{\varepsilon}{L}\right)^{y}\right)\left[C_{\cdot \cdot}^{(a a a) \cdot}\left(\lambda_{a}^{R}\right)^{2}+C_{\cdot \cdot}^{(a b a) \cdot} \mu_{a}^{R} \mu_{a}^{R \dagger}+C_{\cdot \cdot}^{(a c a) \cdot} \mu_{c}^{R} \mu_{c}^{R \dagger}\right], \\
& \left(\frac{\varepsilon}{L}\right)^{-y} \mu_{a}=\mu_{a}^{R}-\frac{1}{y}\left(1-\left(\frac{\varepsilon}{L}\right)^{y}\right)\left[C_{\cdot \cdot}^{(a a b) \cdot} \lambda_{a}^{R} \mu_{a}^{R}+C_{\cdot \cdot}^{(a b b) \cdot} \lambda_{b}^{R} \mu_{a}^{R}\right], \\
& \left(\frac{\varepsilon}{L}\right)^{-y} \mu_{a}^{\dagger}=\mu_{a}^{R \dagger}-\frac{1}{y}\left(1-\left(\frac{\varepsilon}{L}\right)^{y}\right)\left[C_{\cdot \cdot \cdot}^{(a a b) \cdot} \lambda_{a}^{R} \mu_{a}^{R \dagger}+C_{\cdot \cdot}^{(a b b) \cdot} \lambda_{b}^{R} \mu_{a}^{R \dagger}\right],
\end{aligned}
$$

where $b=a+1$ and $c=a-1$. Substituting into $(5.2)$ and using $C_{. .}^{(a b a)} \mathbb{1}_{1}\left\langle\mathbb{1}_{a}\right\rangle=C_{. .}^{(b a b)} \mathbb{1}^{1}\left\langle\mathbb{1}_{b}\right\rangle$ one finds,

$$
\begin{aligned}
& g(\lambda, \mu)=\sum_{a=1}^{n}\left\langle\mathbb{1}_{a}\right\rangle-\pi^{2} y \sum_{a=1}^{n}\left(\lambda_{a}^{R}\right)^{2} C_{. .}^{(a a a)} \mathbb{1}\left\langle\mathbb{1}_{a}\right\rangle-2 \pi^{2} y \sum_{a=1}^{n-1} \mu_{a}^{R} \mu_{a}^{R \dagger} C_{. .}^{(a b a)} \mathbb{1}\left\langle\mathbb{1}_{a}\right\rangle \\
& -\frac{2 \pi^{2}}{3} \sum_{a=1}^{n}\left(\lambda_{a}^{R}\right)^{3} C_{\cdot .}^{(a a a) .} C_{\cdot .}^{(a a a)} \mathbb{1}_{{ }^{\prime}}\left\langle\mathbb{1}_{a}\right\rangle \\
& -2 \pi^{2} \sum_{a=1}^{n-1} \lambda_{a}^{R} \mu_{a}^{R} \mu_{a}^{R \dagger} C_{\cdot .}^{(a a b) .} C_{. \cdot}^{(a b a)} \mathbb{1}\left\langle\mathbb{1}_{a}\right\rangle-2 \pi^{2} \sum_{a=1}^{n-1} \lambda_{b}^{R} \mu_{a}^{R} \mu_{a}^{R \dagger} C_{\cdot .}^{(a b b) .} C_{\cdot .}^{(a b a)} \mathbb{1}^{\prime}\left\langle\mathbb{1}_{a}\right\rangle .
\end{aligned}
$$

In subsequent sections all couplings will be renormalised so we will drop the $R$. We end this section by commenting that the expansions (5.11) are only good for $\lambda<<\lambda^{*}$. However when substituted into physical quantities (e.g. (5.12)), the resulting expressions remain true in the limit $\lambda \rightarrow \lambda^{*}$. However to use (5.11) in this limit, there must exists a $\lambda(L)$ such that $\lim _{L \rightarrow \infty} \lambda(L)=\lambda^{*}$. Such a $\lambda$ was found in section 4.1. 


\subsection{Discussion of g-theorem}

Having calculated $g$, we are almost duty-bound to say a few words about the $g$-theorem of Affleck and Lugwig [3, 2]. Here we note that the $\beta$-functions are gradient flows (c.f. [17]),

$$
\frac{\partial g}{\partial \lambda_{i}}=-\sum_{j} G_{i j} \beta_{j},
$$

where $G$ is a symmetric positive definite matrix with non-zero entries,

$$
\begin{aligned}
& G_{\lambda_{a} \lambda_{a}}=2 \pi^{2} C^{(a a a)} \mathbb{1}_{\text {. }}\left\langle\mathbb{1}_{a}\right\rangle, \\
& G_{\mu_{a} \mu_{a}^{\dagger}}=2 \pi^{2} C_{. .}^{(a b a)} \mathbb{1}_{1}\left\langle\mathbb{1}_{a}\right\rangle=2 \pi^{2} C_{. .}^{(b a b)} \mathbb{1}^{1}\left\langle\mathbb{1}_{b}\right\rangle,
\end{aligned}
$$

and where $b=a+1$. Thus the $g$-theorem,

$$
L \frac{d g}{d L}=-\sum_{i, j} G_{i j} \beta_{i} \beta_{j} \leq 0,
$$

follows from unitarity: The negativity of the contribution from the ordinary fields is clear. For the boundary changing operators, unitarity constrains us to write $\mu=x+i y, \mu^{\dagger}=x-i y$ for real $x$ and $y$ (see section 3 ) and hence

$$
\beta_{\mu} \beta_{\mu^{\dagger}}=\beta_{x}^{2}+\beta_{y}^{2}
$$

\subsection{Evaluation of End-points}

All conformal boundary states in the minimal models may be written as a superposition of Cardy's boundary conditions. We denote Cardy boundary conditions by Greek letters, labeled by a superscript.

$$
\begin{aligned}
\omega & =\oplus_{\ell=1}^{n} \omega^{\ell}, & \alpha & =\oplus_{\ell=1}^{n^{\prime}} \alpha^{\ell}, \\
\omega^{\ell} & =\left(\omega_{1}^{\ell}, \omega_{2}^{\ell}\right)=(r, s+2 \ell-2), & \alpha^{\ell} & =\left(\alpha_{1}^{\ell}, \alpha_{2}^{\ell}\right),
\end{aligned}
$$

where $\omega$ denotes the initial boundary while $\alpha$ will be the final boundary. The boundary entropy of a superposition of boundary states $\omega$, is given by the sum

$$
g_{s u p}^{\omega}=\sum_{\ell} g_{\omega^{\ell}} .
$$

Using (2.5), the object of interest is,

$$
\begin{aligned}
\Delta \ln g & =\ln \left(\frac{g_{\text {sup }}^{\alpha}}{g_{\text {sup }}^{\omega}}\right) \\
& =\ln \left(\frac{\sigma_{\alpha}}{\sigma_{\omega}}\right)+\frac{\pi^{2}}{6}\left(\sum_{\ell} \frac{\omega_{1}^{\ell} \omega_{2}^{\ell}}{\sigma_{\omega}}\left(\left(\omega_{1}^{\ell}\right)^{2}+\left(\omega_{2}^{\ell}\right)^{2}\right)-\sum_{\ell} \frac{\alpha_{1}^{\ell} \alpha_{2}^{\ell}}{\sigma_{\alpha}}\left(\left(\alpha_{1}^{\ell}\right)^{2}+\left(\alpha_{2}^{\ell}\right)^{2}\right)\right)(m+1)^{-2} \\
& +\frac{\pi^{2}}{3}\left(\sum_{\ell} \frac{\omega_{1}^{\ell} \omega_{2}^{\ell}}{\sigma_{\omega}}\left(\omega_{1}^{\ell}\right)^{2}-\sum_{\ell} \frac{\alpha_{1}^{\ell} \alpha_{2}^{\ell}}{\sigma_{\alpha}}\left(\left(\alpha_{1}^{\ell}\right)^{2}\right)(m+1)^{-3}+O\left((m+1)^{-4}\right)\right.
\end{aligned}
$$


where

$$
\sigma_{\omega}=\sum_{\ell} \omega_{1}^{\ell} \omega_{2}^{\ell}
$$

From the perturbative calculation, this is given by (5.12),

$$
\Delta \ln g\left(\lambda^{*}, \mu^{*}\right)=\ln \left(\frac{g\left(\lambda^{*}, \mu^{*}\right)}{g_{\text {sup }}^{\omega}}\right) .
$$

Without loss of generality, we can assume all the boundary changing couplings are non-zero. Inserting values for the structure constants, the fixed points found in section 3.2.1, and $g_{\text {sup }}^{\omega}$ one finds the rather simple result,

$$
\begin{aligned}
& \Delta \ln g\left(\lambda^{*(1)}, \mu^{*}\right)=-\frac{\pi^{2}}{3} \frac{(s+n-2)(s+n)}{(m+1)^{3}}, \\
& \Delta \ln g\left(\lambda^{*(2)}, \mu^{*}\right)=-\frac{\pi^{2}}{3} \frac{(n-1)(n+1)}{(m+1)^{3}},
\end{aligned}
$$

Equating this with (5.21) provides the following set of polynomial equations to be solved over the integers,

$$
\begin{aligned}
\sigma_{\omega} & =\sigma_{\alpha}, \\
\sum_{\ell} \frac{\omega_{1}^{\ell} \omega_{2}^{\ell}}{\sigma_{\omega}}\left(\left(\omega_{1}^{\ell}\right)^{2}+\left(\omega_{2}^{\ell}\right)^{2}\right) & =\sum_{\ell} \frac{\alpha_{1}^{\ell} \alpha_{2}^{\ell}}{\sigma_{\alpha}}\left(\left(\alpha_{1}^{\ell}\right)^{2}+\left(\alpha_{2}^{\ell}\right)^{2}\right), \\
\sum_{\ell} \frac{\alpha_{1}^{\ell} \alpha_{2}^{\ell}}{\sigma_{\alpha}}\left(\left(\alpha_{1}^{\ell}\right)^{2}-\sum_{\ell} \frac{\omega_{1}^{\ell} \omega_{2}^{\ell}}{\sigma_{\omega}}\left(\omega_{1}^{\ell}\right)^{2}\right. & = \begin{cases}(s+n-2)(s+n) & \text { for solution 1, } \\
(n-1)(n+1) & \text { for solution 2. }\end{cases}
\end{aligned}
$$

As in [14], these equations generally have many solutions, however a generic solution does appear. For solution 1 we have,

$$
\bigoplus_{i=1}^{\min }\{r, s+n-1\}(r+s+n-2 i, n)
$$

while for solution 2 ,

$$
\bigoplus_{i=1}^{\min \{r, n\}}(r+n+1-2 i, s+n-1)
$$

The calculation of $g$ is ambiguous because of the symmetries $g_{(r, s)}=g_{(m-r, s)}=g_{(r, m+1-s)}$. One may remove this uncertainty by simply calculating the one point functions of bulk fields to zeroth order in perturbation theory.

We complete this section with our example of $\omega=(1,1) \oplus(1,3) \oplus(1,5)$. In section 3.2.1 we found nine fixed points for the system, we now identify the boundary theories associated to these points, 


\begin{tabular}{ll}
\hline $\begin{array}{l}\text { Fixed Point } \\
\left\{\lambda_{2}, \lambda_{3}, \mu_{1} \mu_{1}^{\dagger}, \mu_{2} \mu_{2}^{\dagger}\right\}\end{array}$ & Boundary Theory \\
\hline$\{0,0,0,0\}$ & $(1,1) \oplus(1,3) \oplus(1,5)$ \\
$\{-y, 0,0,0\}$ & $(1,1) \oplus(3,1) \oplus(1,5)$ \\
$\{0,-\sqrt{3} y, 0,0\}$ & $(1,1) \oplus(1,3) \oplus(5,1)$ \\
$\{-y,-\sqrt{3} y, 0,0\}$ & $(1,1) \oplus(3,1) \oplus(5,1)$ \\
$\left\{-\frac{1}{2} y,-\frac{\sqrt{3}}{2} y, y^{2}, \frac{5}{12} y^{2}\right\}$ & $(3,3)$ \\
$\left\{-\frac{1}{2} y, 0, \frac{3}{8} y^{2}, 0\right\}$ & $(2,2) \oplus(1,5)$ \\
$\left\{-\frac{5}{4} y,-\frac{3 \sqrt{3}}{4} y, 0, \frac{5}{16} y^{2}\right\}$ & $(1,1) \oplus(2,4)$ \\
$\left\{\frac{1}{4} y,-\frac{\sqrt{3}}{4} y, 0, \frac{5}{16} y^{2}\right\}$ & $(1,1) \oplus(4,2)$ \\
$\left\{-\frac{1}{2} y,-\sqrt{3} y, \frac{3}{8} y^{2}, 0\right\}$ & $(2,2) \oplus(5,1)$ \\
\hline
\end{tabular}

Table 国: The Identification of the fixed points of the $(1,1) \oplus(1,3) \oplus(1,5)$ system.

\section{A Connection with Lattice theories}

With the wealth of fixed points observed in these systems, it would be helpful to find a nice way of organising everything. In this section, we provide a simple set of rules which we claim, describe the space of flows for an arbitrary Cardy boundary condition.

There is an intriguing connection between the flows found here and the lattice representation of conformal boundary conditions found in [4] which we will now explain. In [4], each boundary condition was shown to be related to a set of integrable lattice boundary weights which when taken to the continuum limit, returned the boundary theory. As part of their construction, the authors associate to each Cardy boundary condition in the minimal model $M_{m, m+1}$, a subgraph of (in our case) the Dynkin diagram $A_{m}$. It is this subgraph that is of interest to us.

Let us define fused adjacency matrix $F_{a b}^{r}$ as follows,

$F_{a b}^{r}=N(m+1)_{r a}{ }^{b}=\left\{\begin{array}{lc}1 & a+b+r \text { odd, }|a-b| \leq r-1 \text { and } r+1 \leq a+b \leq 2 m-r+1, \\ 0 & \text { otherwise. }\end{array}\right.$

$F_{a b}^{r}$ is symmetric in all three indices.

We label the nodes of $A_{m}$ by $1,2, \ldots, m$ then the subgraph associated to the Cardy boundary condition $(r, s)$ is the set of linked nodes,

$$
\left\{(i, i \pm 1): F_{i s}^{r} F_{i \pm 1, s}^{r+1}>0\right\} .
$$

Distilling this for the case at hand, we see the $(r, s)$ boundary condition in the limit of large $m$, is represented by the subgraphs,

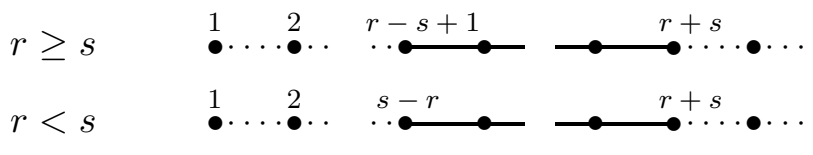


A first indication that these diagrams have relevance to boundary perturbations is the observation that the number of links in the diagram is equal to the number of relevant operators on the boundary (The proof is contained in appendix A.4).

We now introduce our diagrammatic rules for the flows. Flows will correspond to projections onto subgraphs. Assign an orientation to each link in the graph whereby the link connected to node $r+s$ is negative and contiguous links have opposite orientation, for example for $(3,3)$,

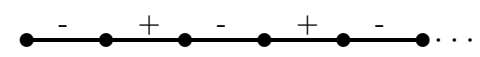

A perturbative flow then corresponds to deleting a set of positive links,

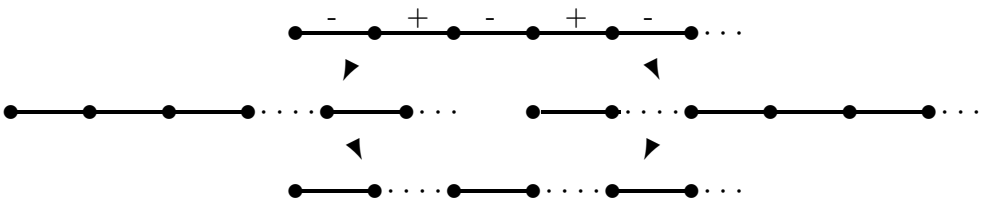

We now turn to our boundary changing flows. For this we consider a superposition of $(1, s)$ boundaries, and represent the boundary changing operators as vertical links joining the end nodes of the boundaries, for example $(1,1) \oplus(1,3) \oplus(1.5)$,

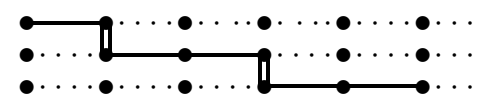

Boundary changing flows then correspond to deleting these links and fusing the subgraphs together,

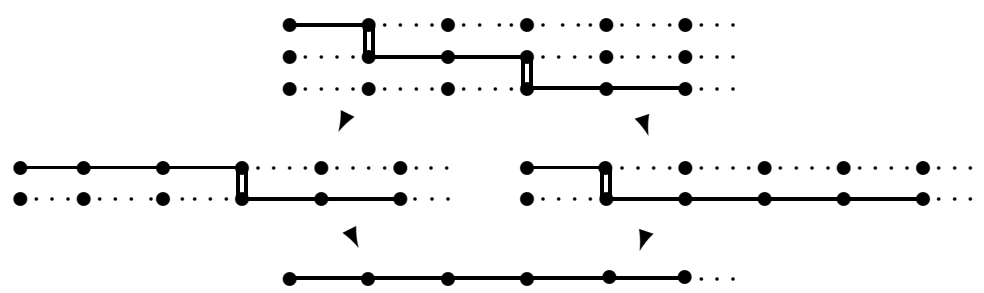

One can see that in general, all the possible perturbative end points of flows from a superposition of $(1, s)$ are represented in the subgraphs generated by successive deletions and fusings. Furthermore, it is our belief that the space of possible perturbative endpoints is represented by this procedure: A perturbative flow from $a \rightarrow b$ exists if and only if the graph of $b$ is a subgraph of $a$ obtained by fusing and/or by deleting positive links.

As an example, we consider the case of $(1, p-1) \oplus(1, p+1)$. Using the above rules we construct the following diagram.

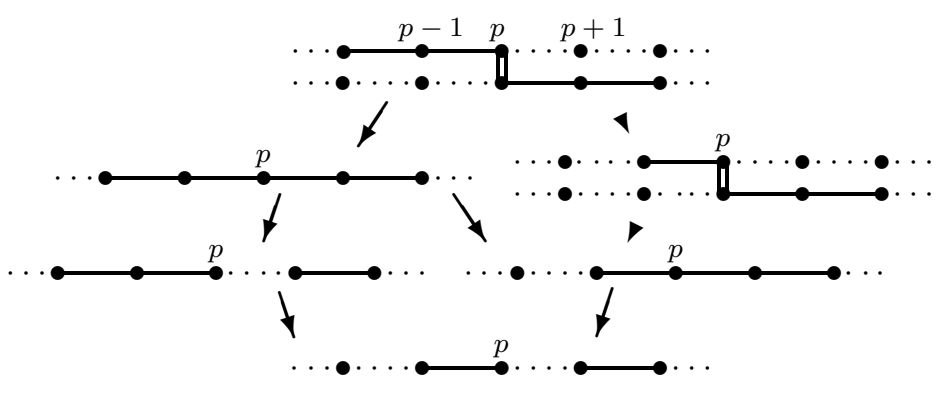




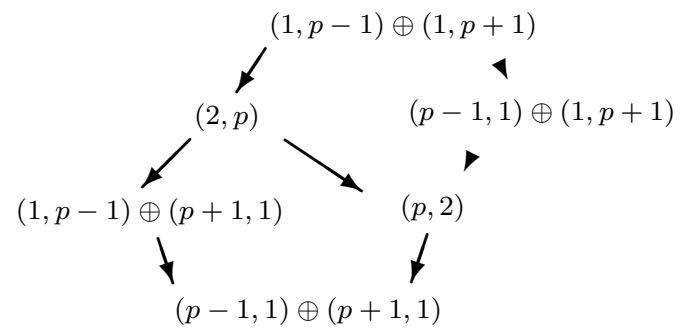

Thus we predict that the $(2, p)$ boundary contains flows to $(p, 2)$ and $(1, p-1) \oplus(p+1,1)$ while $(p-1,1) \oplus(1, p+1)$ contains a $\phi_{24}$ boundary changing flow to $(p, 2)$ in agreement with the numerical studies of section 4.2 .

Maybe we can go further. TCSA results [16] suggest that in the non-perturbative direction the $\phi_{13}$ perturbation of a Cardy boundary condition flowed to,

$$
(r, s) \rightarrow \oplus_{i=1}^{\min \{r, s-1\}}(r+s-2 i, 1)
$$

see also [14]. In our diagrammatic rules, this corresponds to deleting all the negative links. Furthermore in [11, 10], it was observed that perturbations by $\phi_{r r}$ fields can act as "projectors", flowing onto one of the boundaries $(1, s+2 t-2)$ or $(s+2 t-2,1)$ for some $t=1, \ldots, n$. In our diagrammatic rules, this can again be represented by projecting onto a subgraph.

All in all we feel the evidence is strong that the space of end points of general perturbations of a $(r, s)$ boundary condition are described succinctly by the subgraphs of its associated graph.

\section{Conclusions}

In conclusion, we have studied perturbations of superpositions of Cardy boundary conditions within perturbation theory. We showed how to study perturbations by $\phi_{r, r+2}$ fields on general superpositions of boundary conditions. For two examples, boundary changing $\phi_{13}$ operators and perturbations by $\phi_{35}$, we explicitly constructed the space of perturbative flows and studied the fixed points. We noted that by a suitable choice of superposition of $(1, s)$ boundary conditions, one could construct an arbitrary Cardy boundary condition and study its space of flows from the field theory on the superposition. Furthermore, we showed that to lowest order in the examples considered, the $\beta$-functions describing both the superposition and the Cardy boundary were identical after a redefinition of fields. We then went on and conjectured a diagrammatic representation for the space of renormalisation group fixed points of a Cardy boundary condition.

A lot of work still needs to be done.

- The diagrammatic rules need to be proven for the perturbative flows as well as more checks carried out in the non-perturbative case.

- Can one represent more general boundary changing flows diagrammatically? In particular is there a lattice realisation for a superposition of boundary conditions? Does there exist a lattice realisation of these flows? 
- Are any of these flows integrable?

- What of $\phi_{r, r \pm 1}$ ? These fields have a character all of their own that has yet to be investigated.

- Lattice realisations of the D and E Series minimal models are also available, are their boundary flows also represented in such a manor?

Note added. After the completion of this work, we became aware of [9] where some of the results of this paper were found by other means.

\section{Acknowledgments}

The author thanks Ingo Runkel and James Drummond for helpful discussions and support. He also thanks S. Fredenhagen, A. Recknagel and V. Schomerus for comments and correspondance as well as IPAM-UCLA for their hospitality during the latter stages of this work. Finally, he would like to thank Gerard Watts whose help has been indispensable, thanks. Supported by an EPSRC studentship.

\section{Appendices}

\section{A.1 Structure Constants for $\phi_{13}$}

Due to the constraint $C_{i i}^{(a b a)} \mathbb{1}_{1}\left\langle\mathbb{1}_{a}\right\rangle=C_{i i}^{(b a b)} \mathbb{1}_{i}\left\langle\mathbb{1}_{b}\right\rangle$, it is not possible to set both $C_{i i}^{(a b a) \mathbb{1}}$ and $C_{i i}^{(b a b)} \mathbb{1}$ equal to one. Instead we define an ordering on pairs $a_{i}=\left(r_{i}, s_{i}\right)$ whereby $a_{1}<a_{2}$ if $r_{1}<r_{2}$, and if $r_{1}=r_{2}$ then $a_{1}<a_{2}$ if $s_{1}<s_{2}$. We define,

$$
C_{i i}^{(a b a)} \mathbb{1}=\left\{\begin{array}{lr}
1 & \text { if } a \leq b, \\
\left\langle\mathbb{1}_{b}\right\rangle /\left\langle\mathbb{1}_{a}\right\rangle & \text { otherwise. }
\end{array}\right.
$$

The leading behaviours of the structure constants for $\phi_{13}$ fields in the $m \rightarrow \infty$ limit are as follows,

$$
\begin{aligned}
& a=(r, 1), b=(r, 3) \\
& C_{. \cdot}^{(a a a) \cdot}=C_{. \cdot}^{(a b a) \cdot}=C_{. \cdot}^{(a a b) \cdot}=C_{. \cdot}^{(a a a) \mathbb{1}}=0, \\
& C_{. .}^{(b b b) .}=1, \quad C_{. \cdot}^{(b a b) .}=\frac{2}{3}, \quad C_{. .}^{(a b b) .}=2, \\
& C_{. .}^{(b b b) \mathbb{1}}=1, \quad C_{. .}^{(a b a) \mathbb{1}}=1, \quad C_{. .}^{(b a b) \mathbb{1}}=\frac{1}{3} . \\
& a=(r, s), b=(r, s+2), s \neq 1 \\
& C^{(a a a) .}=\sqrt{\frac{8}{s^{2}-1}}, \quad \quad C_{\cdot .}^{(a a b) .}=C_{\cdot .}^{(b a a) .}=C_{\cdot .}^{(a b a) .}=-\sqrt{2 \frac{s-1}{s+1}}, \\
& C_{\cdot \cdot}^{(b a b) .}=\frac{s}{s+2} \sqrt{2 \frac{s+3}{s+1}}, \quad C_{\cdot \cdot}^{(b b a) .}=C_{\cdot \cdot}^{(a b b) .}=\sqrt{2 \frac{s+3}{s+1}},
\end{aligned}
$$




$$
C_{\cdot \cdot}^{(a a a) \mathbb{1}}=1, \quad C_{. \cdot}^{(a b a)} \mathbb{1}=1, \quad C_{. \cdot}^{(b a b) \mathbb{1}}=\frac{s}{s+2},
$$

where we have used a dot to denote $\phi_{13}$.

\section{A.2 Structure Constants for the $(2, p)$ Boundary}

All fields on the $(2, p)$ boundary are normalised so that $C_{i i}^{(. .)} \mathbb{1}=1$. In the case of $L_{-1} \phi_{33}$ we have,

$$
L_{-1} \phi_{33}(x) L_{-1} \phi_{33}(0)=2 h_{33}\left(1+2 h_{33}\right) x^{-2-2 h_{33}} \mathbb{1}+\ldots
$$

To leading order $2 h_{33}\left(1+2 h_{33}\right)=\left(\frac{2}{m+1}\right)^{2}$ and so we normalise,

$$
d_{3}=\frac{m+1}{2} L_{-1} \phi_{33} .
$$

The structure constants for the $(2, p)$ boundary to leading order as $m \rightarrow \infty$ are, with $\phi=\phi_{13}, \quad \tilde{\phi}=\phi_{31}, \quad c_{3}=\phi_{33}, \quad d_{3}=\frac{m+1}{2} L_{-1} \phi_{33}, \quad \psi=\phi_{35}$,

$$
\begin{aligned}
& C_{\phi \phi}^{(. .) \phi}=\sqrt{\frac{8}{p^{2}-1}}, \\
& C_{\tilde{\phi} \tilde{\phi}}^{(. .) \tilde{\phi}}=\sqrt{\frac{8}{3}}, \quad \quad C_{\phi \tilde{\phi}}^{(\ldots) c}=-\sqrt{\frac{1}{3}}, \\
& C_{c c}^{(. .) c}=\sqrt{\frac{4}{p^{2}-1}}, \\
& C_{\phi \psi}^{(. .) \psi}=3 \sqrt{\frac{2}{p^{2}-1}}, \quad C_{\tilde{\phi} \psi}^{(. .) \psi}=-\sqrt{\frac{2}{3}}, \\
& C_{\phi c}^{(\cdots) \psi}=-\sqrt{\frac{2}{3} \frac{p^{2}-4}{p^{2}-1}} \\
& C_{c \psi}^{(. .) \psi}=\sqrt{\frac{9}{p^{2}-1}}, \quad C_{\psi \psi}^{(. .) \psi}=\left(p^{2}-16\right) \sqrt{\frac{4}{3} \frac{1}{\left(p^{2}-4\right)\left(p^{2}-1\right)}}, \\
& C_{\phi c}^{(. .) c}=-\sqrt{\frac{8}{p^{2}-1}} \cdot \frac{1}{m+1}, \quad C_{\tilde{\phi} c}^{(\ldots) c}=\sqrt{\frac{8}{3}} \cdot \frac{1}{m+1}, \quad C_{c c}^{(. .) \psi}=-\sqrt{\frac{4}{3} \frac{p^{2}-4}{p^{2}-1}} \cdot \frac{1}{m+1},
\end{aligned}
$$

where dots denote the $(2, p)$ boundary. The structure constants (A.6) are totally symmetric in all indices.

The structure constants involving an odd number of descendent fields $d_{3}$ are all antisymmetric in the lower two indices and so do not contribute to the $\beta$-functions. The remaining are symmetric and take the form,

$$
\begin{aligned}
& C_{\phi d}^{(. .) d}=-C_{d d}^{(. .) \phi}=\sqrt{\frac{2}{p^{2}-1}}, \\
& C_{\tilde{\phi} d}^{(. .) d}=-C_{d d}^{(. .) \tilde{\phi}}=\sqrt{\frac{2}{3}}, \\
& C_{\psi d}^{(. .) d}=-C_{d d}^{(. .) \psi}=\sqrt{\frac{4}{3} \frac{p^{2}-4}{p^{2}-1}} .
\end{aligned}
$$




\section{A.3 Solving the $\beta$-functions}

We will attempt to find a solution to the $\beta$-function equations,

$$
L \frac{d \lambda_{i}}{d L}=y_{i} \lambda_{i}+\sum_{j, k} C_{j k}^{i} \lambda_{j} \lambda_{k}, \quad \lambda_{i}^{b}=\lambda_{i}(\varepsilon)
$$

of the form

$$
\lambda_{i}^{b}=\sum_{j} a_{j}^{i}(L) \lambda_{j}(L)+\sum_{j, k} b_{j k}^{i}(L) \lambda_{j}(L) \lambda_{k}(L) .
$$

First differentiate with respect to $L$ and substitute in expression for the $\beta$-function.

$$
0=\sum_{j}\left\{L \frac{d a_{j}^{i}}{d L} \lambda_{j}+a_{j}^{i}\left(y_{j} \lambda_{j}+\sum_{k, l} C_{k l}{ }^{j} \lambda_{k} \lambda_{l}\right)\right\}+\sum_{j, k}\left\{L \frac{d b_{j k}^{i}}{d L} \lambda_{j} \lambda_{k}+\left(y_{j}+y_{k}\right) b_{j k}^{i} \lambda_{j} \lambda_{k}\right\} .
$$

Equating coefficients one obtains the following ODE's,

$$
\begin{aligned}
& 0=L \frac{d a_{j}^{i}}{d L}+y_{j} a_{j}^{i}, \\
& 0=L \frac{d b_{j k}^{i}}{d L}+\left(y_{j}+y_{k}\right) b_{j k}^{i}+\sum_{l} C_{j k}^{l} a_{l}^{i},
\end{aligned}
$$

which using the boundary condition become,

$$
\begin{aligned}
a_{j}^{i} & =\delta_{j}^{i}\left(\frac{\varepsilon}{L}\right)^{y_{j}}, \\
b_{j k}^{i} & =\left\{\begin{array}{ll}
\frac{1}{y_{i}-y_{j}-y_{k}} C_{j k}{ }^{i}\left[\left(\frac{\varepsilon}{L}\right)^{y_{i}}-\left(\frac{\varepsilon}{L}\right)^{y_{j}+y_{k}}\right] & \text { if } y_{i} \neq y_{j}+y_{k} \\
C_{j k}{ }^{i}\left(\frac{\varepsilon}{L}\right)^{y_{j}+y_{k}} \ln \left(\frac{\varepsilon}{L}\right) & \text { if } y_{i}=y_{j}+y_{k}
\end{array} .\right.
\end{aligned}
$$

Hence,

$$
\begin{gathered}
\left(\frac{\varepsilon}{L}\right)^{-y_{i}} \lambda_{i}^{b}=\lambda_{i}(L)+\sum_{\substack{j k \\
y_{i} \neq y_{j}+y_{k}}}\left(\frac{1}{y_{i}-y_{j}-y_{k}} C_{j k}{ }^{i}\left(1-\left(\frac{\varepsilon}{L}\right)^{-y_{i}+y_{j}+y_{k}}\right)\right) \lambda_{j}(L) \lambda_{k}(L) \\
+\sum_{\substack{j k \\
y_{i}=y_{j}+y_{k}}} C_{j k}{ }^{i} \ln \left(\frac{\varepsilon}{L}\right) \lambda_{j}(L) \lambda_{k}(L)
\end{gathered}
$$

\section{A.4 Links and Relevant Operators}

In this section we show that the number of links in a diagram of Behrend and Pearce is equal to the number of relevant operators on the appropriate conformal boundary condition. Not only is this true for the A-series mentioned in the text, but also for the $\mathrm{D}$ and $\mathrm{E}$ models too.

For the details of statements made in this section, we refer the reader to [5] and [4]. 
In the notation of [5], we look at the model $M\left(A_{m-1}, G\right)$ with $G$ an ADE graph with Coexter number $g=m+1$. For the A-Series $G=A_{m}$. Note that we will not consider the unitary models with $g=m-1$ because as of this writing, they lack a lattice realisation with suitable boundary weights.

Let $G_{a b}$ be the adjacency matrix of $G$ which defines the fused adjacency matrix $F_{a b}^{r}$,

$$
F_{a b}^{r+1}=\sum_{c} G_{a c} F_{c b}^{r}-F_{a b}^{r-1}, \quad F_{a b}^{1}=\mathbb{1}, \quad F_{a b}^{2}=G_{a b},
$$

The $F^{r}$ are symmetric matrices with positive integer entries and $F^{m+1}=0$. In the case $G=A_{m}, F_{a b}^{r}$ is given by equation (6.1).

The ADE graph $G$ is bicolourable; that is to say, one may assign to each node $a \in G$, a parity $\pi_{a} \in\{-1,1\}$ such that adjacent nodes have opposite parity, $G_{a b}>0 \Longrightarrow \pi_{a} \pi_{b}=-1$. This extends to $F^{r}$ :

$$
F_{a b}^{r}>0 \Longrightarrow \pi_{a} \pi_{b}=(-1)^{r+1}
$$

As matrices, the $F^{r}$ form a representation of the fusion algebra,

$$
F^{r} F^{s}=\sum_{t} N(m+1)_{r s}^{t} F^{t}
$$

where $N(m)_{r s}^{t}$ is given in (2.4). One may also write the boundary spectrum of a general minimal model in terms of these matrices [5] (c.f. (2.3)),

$$
n_{(r, s)\left(r^{\prime}, s^{\prime}\right)}^{\left(r^{\prime \prime}, s^{\prime \prime}\right)}=N(m)_{r, r^{\prime}} r^{\prime \prime} F_{s^{\prime} s^{\prime \prime}}^{s}+N(m)_{m-r, r^{\prime}} r^{\prime \prime} F_{s^{\prime} s^{\prime \prime}}^{m+1-s}
$$

Following [ [A], we label the nodes of $G$ by $1,2, \ldots, n$ then the subgraph associated to the Cardy boundary condition $(r, s)$ has the adjacency matrix,

$$
B(r, s)_{a b}=F_{s a}^{r} G_{a b} F_{b s}^{r+1} .
$$

We now turn to our theorem. From (2.1), relevant fields in a boundary minimal model have the form $\phi_{r, r+t}$ for $t \in\{-1,0,1,2\}$. Using (A.19) we find the number of relevant fields is given by,

$$
\text { \# rel. fields on }(r, s)=\sum_{\substack{k \\ t \in\{-1,0,1,2\}}} n_{(k, k+t)(r, s)}^{(r, s)}=\sum_{k} N(m)_{k r}^{r}\left(F_{s s}^{k}+F_{s s}^{k+2}\right) \text {, }
$$

where the sum over $k$ in the first expression is chosen as to avoid over counting due to the identification $(r, s) \leftrightarrow(m-r, m+1-s)$. In obtaining the second expression we have used the bicolourability of $G$. 
On the other hand, counting the number of links in this subgraph with multiplicities we find,

$$
\# \text { links }=\sum_{a, b} F_{s a}^{r} G_{a b} F_{b s}^{r+1}
$$

As an aside, there is no over counting in (A.22) because the bicolourability of $G$ implies that $(a, b) \in B \Longrightarrow(b, a) \notin B$. From (A.22), (A.16) and (A.18),

$$
\# \text { links }=\sum_{a} F_{s a}^{r}\left(F_{a s}^{r}+F_{a s}^{r+2}\right)=\sum_{k} N(m+1)_{r r}{ }^{k} F_{s s}^{k}+N(m+1)_{r, r+2}{ }^{k} F_{s s}^{k} .
$$

To make the connection with (A.21), we consider each term in turn. Using the explicit realisation of $N(m),(2.4)$, we write,

$$
\begin{aligned}
\sum_{k} N(m+1)_{r r}^{k} F_{s s}^{k} & =\sum_{k} N(m)_{r r}^{k} F_{s s}^{k}+Q_{1}, \\
Q_{1} & = \begin{cases}F_{s s}^{2(m-r)+1} & \text { when } \quad r \geq \frac{1}{2}(m+1), \\
0 & \text { otherwise } .\end{cases}
\end{aligned}
$$

and,

$$
\begin{aligned}
\sum_{k} N(m+1)_{r, r+2}{ }^{k} F_{s s}^{k} & =\sum_{k} N(m)_{r, r}{ }^{k} F_{s s}^{k+2}-Q_{2}, \\
Q_{2} & = \begin{cases}F_{s s}^{2(m-r)+1} & \text { when } \quad r \geq \frac{1}{2} m, \\
0 & \text { otherwise }\end{cases}
\end{aligned}
$$

Now, $Q_{1}$ and $Q_{2}$ cancel except when $r=\frac{1}{2} m, Q_{2}=F^{m+1}=0$. Putting this all together,

$$
\# \text { links }=\sum_{k} N(m)_{r r}^{k}\left(F_{s s}^{k}+F_{s s}^{k+2}\right)
$$

which is equal to (A.21) by the cyclic nature of $N(m)$.

\section{References}

[1] I. Affleck, Edge Critical Behaviour of the 2-Dimensional Tri-critical Ising Model, J. Phys. A 33 (2000) 6473 arXiv:cond-mat/0005286.

[2] I. Affleck and A. W. Ludwig, Exact conformal field theory results on the multichannel Kondo effect: Single fermion Green's function, selfenergy and resistivity, Phys. Rev. B48 (1993) 7297

[3] I. Affleck and A. W. Ludwig, Universal noninteger 'ground state degeneracy' in critical quantum systems, Phys. Rev. Lett. 67 (1991) 161. 
[4] R. E. Behrend and P. A. Pearce, Integrable and conformal boundary conditions for sl(2) A-D-E lattice models and unitary minimal conformal field theories, arXiv:hepth/0006094.

[5] R. E. Behrend, P. A. Pearce, V. B. Petkova and J. Zuber, Boundary conditions in rational conformal field theories, Nucl. Phys. B 570 (2000) 525 [Nucl. Phys. B 579 (2000) 707] arXiv:hep-th/9908036.

[6] J. Cardy, Scaling and renormalization in statistical physics, Cambridge, UK: Univ. Pr. (1996) 238 p. (Cambridge lecture notes in physics: 3).

[7] J. L. Cardy, Boundary Conditions, Fusion Rules And The Verlinde Formula, Nucl. Phys. B324 (1989) 581.

[8] J. L. Cardy, Conformal invariance and statistiacal mechanics, Lectures given at Les Houches (1988)

[9] S. Fredengagen and V. Schomerus, D-Branes in Coset Models, arXiv:hep-th/0111189.

[10] K. Graham, I. Runkel and G. M. T. Watts, Minimal model boundary flows and $c=1$ CFT, Nucl. Phys. B 608 (2001) 527 [arXiv:hep-th/0101187].

[11] K. Graham, I. Runkel and G. M. T. Watts, Renormalisation group flows of boundary theories, Talk by GMTW at "Non-perturbative Quantum Effects 2000", PRHEPtmr2000/040 arXiv:hep-th/0010082.

[12] K. Graham and G. M. T. Watts, In Preparation.

[13] For example: J. Polchinski, String Theory. Vol. 1: An Introduction To The Bosonic String, Cambridge, UK: Univ. Pr. (1998) 402 p.

[14] A. Recknagel, D. Roggenkamp and V. Schomerus, On relevant boundary perturbations of unitary minimal models, Nucl. Phys. B 588 (2000) 552 [arXiv:hep-th/0003110.

[15] I. Runkel, Boundary structure constants for the A-series Virasoro minimal models, Nucl. Phys. B549 (1999) 563 arXiv:hep-th/9811178].

[16] G. M. T. Watts, unpublished.

[17] A. B. Zamolodchikov, Renormalization Group And Perturbation Theory Near Fixed Points In Two-Dimensional Field Theory, Sov. J. Nucl. Phys. 46 (1987) 1090 [Yad. Fiz. 46 (1987) 1819]. 